\title{
Acid resistance of calcium aluminate cement-fly ash F blends
}

\section{Tatiana Pyatina}

Sustainable Energy Technologies, Brookhaven National Laboratory, Upton, NY, USA (corresponding author: tpyatina@bnl.gov)
Toshifumi Sugama

Sustainable Energy Technologies, Brookhaven National Laboratory, Upton, NY, USA

The short-term resistance to sulfuric acid at $90^{\circ} \mathrm{C}$ of four calcium aluminate cement (CAC)-fly ash class $F$ (FAF) blends activated with sodium metasilicate (thermal shock resistant cements (TSRCs)), cured at $300^{\circ} \mathrm{C}$, was compared to that of a calcium phosphate cement (CPC) (CAC-FAF blend activated with sodium hexametaphosphate) and a Portland cement class G/silica blend. The mechanical properties and compositions of the acid-exposed samples were evaluated by measuring their compressive strength and by means of $x$-ray diffraction, $\mu$ EDX (energy-dispersive $x$-ray spectrometry), thermogravimetric and Fourier transform infrared analyses. All calcium-containing hydrates were sensitive to the conditions of acid exposure. In the TSRC blends, these hydrates included hydrogrossular, feldspar family minerals and zeolites; in CPC, feldspar minerals and phosphate phases; and in the class G/silica blend, portlandite and tobermorite. Crystalline calcium sulfates formed in the acid-exposed surfaces with the exception of the most aluminium-rich TSRC samples where only potassium(sodium) aluminium sulfate, alunite, was detected. This sample underwent the least changes in weight, compressive strength and had the lowest sulfur permeation into the sample core. Calcium sulfates precipitated on sample surfaces limited sulfur penetration into the core of calcium-rich TSRC, CPC and G/silica blends.

\section{Introduction}

In geothermal wells, strongly acidic environments come from the dissolution of hydrogen sulfide $\left(\mathrm{H}_{2} \mathrm{~S}\right)$ with the formation of sulfuric acid $\left(\mathrm{H}_{2} \mathrm{SO}_{4}\right)$. This leads to a very low $\mathrm{pH}(<1)$ hot-acid attack on the cement sheath (Alexander et al., 2013; Lichti and Yanagisawa, 2015; Villasenor and Vicedo, 2010). The degradation of Portland cement by acid proceeds through decalcification of portlandite followed by calcium loss from calcium-silicate hydrate phases at a $\mathrm{pH}$ below about $12 \cdot 4$, causing an increase in porosity and decrease in mechanical strength (Beddoe and Dorner, 2005; Glasser et al., 2008; Gutberlet et al., 2015; Kudowski, 2004; Puertas et al., 2012; Zivica, 2004). The acid diffusivity through the calcium-depleted hydrates is of an order of magnitude higher than that through the original material (Bentz and Garboczi, 1992). The calcium is replaced by acid protons linking the silicate chains and leaving just a silica residue at a $\mathrm{pH}$ below about 2 (Beddoe and Dorner, 2005; Chen et al., 2004; Haga et al., 2002). In the geothermal industry, silica polymerisation caused by low-pH environments is a known problem (Lichti and Yanagisawa, 2015). The precipitation of calcium-acid anions reaction products may either slow down acid attack (Zivica and Bajza, 2002) or enhance erosion and compromise the matrix integrity (Larreur-Cayol et al., 2011).

The iron- and aluminium-containing hydrates of Portland cement dissolve slower and at lower $\mathrm{pH}$ values than calcium hydrates (Beddoe and Dorner, 2005), so the addition of supplementary cementitious materials (SCMs) to Portland cement has been tested and shown to improve the cement's acid resistance by lowering the calcium content and decreasing porosity (Al-Akhras, 2006; Bakharev 2005; Bakharev et al., 2002; Lee et al., 2005; Ouyang et al., 1988). In the case of attack by sulfate ions, SCMs also help to preserve matrix integrity since they do not form expanding reaction products. Using solely alkali-activated SCMs (Chindaprasirt et al., 2014) and incorporating them in reactive aluminium (Kandasamy and Shehata, 2014) has been reported to decrease ion diffusion and help enhance sulfate resistance. However, the gel hydration products of cements with alkali-activated SCMs such as fly ash undergo dealumination of aluminosilcates with the formation of the same final amorphous silica gel (Allahverdi and Skvara, 2001; Allahverdi and Skvara, 2005; Donatello et al., 2013). Geopolymers with crystalline zeolites in the structure formed from activated fly ash were also shown to lose strength at $95^{\circ} \mathrm{C}$ in acidic media (Bakharev, 2005).

Calcium aluminate cement (CAC) has the advantage of a lower calcium content than Portland cement. The nature of its hydrates and the products of sulfuric acid reactions also help to slow down the degradation of CAC with sulfuric acid at $\mathrm{pH}$ levels of above $\sim 3$ (Lamberet et al., 2008). At lower pH levels, however - although slowly - aluminium hydroxide dissolves in acid and eventually the acid degradation of cement proceeds. 
To the authors' knowledge the performance of hightemperature hydration products of blends based on CAC and SCMs in strong acids is not known.

This paper focuses on the short-time changes in the hydrate phase compositions and morphologies of CAC blends with sodium-metasilicate-activated fly ash class F (FAF) during an attack by sulfuric acid at $90^{\circ} \mathrm{C}$. These blends were developed as thermal shock resistant cements (TSRCs) for geothermal wells (Sugama et al., 2012). A Portland cement class G/silica $70 / 30 \mathrm{wt} \%$ blend was tested as a baseline. A CAC blend with FAF activated with sodium hexametaphosphate (calcium phosphate cement (CPC)), developed previously for applications in geothermal wells and tested for acid resistance (Sugama, 1997; Sugama et al., 1999), was also investigated for comparison. To evaluate the role of calcium content, the studied formulations contained two types of CAC - low-calcium CAC\#80 in blends TSRC-1 and TSRC-3 and high-calcium CAC\#51 in blends TSRC-2 and TSRC-4 (Tables 1 and 2). To assess the effect of the SCM on acid resistance, the fly ash content of the blends was also varied, between $40 \mathrm{wt} \%$ (TSRC-1 and TSRC-2) and $60 \mathrm{wt} \%$ (TSRC-3 and TSRC-4). The $\mathrm{pH}$ value was chosen based on cement applications in geothermal wells with highly acidic environments where not only calcium but also ferrite and aluminate hydrates of Portland cement become soluble (Beddoe and Dorner, 2005). It was of interest to assess how the aluminium solubility at this $\mathrm{pH}$ affects the damaged layer composition and sample integrity, and it also allowed acceleration of the acid attack.

\section{Experimental procedure}

Table 1 lists the mix proportions of the tested blends and Table 2 gives the oxide compositions of their components determined by energy-dispersive x-ray spectrometry (EDX). The CACs for use in the TSRC blends were supplied by Kerneos Inc. The class $G$ cement used was a Dyckerhoff North type.

Sodium metasilicate granular powder (supplied by PQ Corporation under the trade name Metso Beads 2048) was used as the alkali activator of FAF in the TSRC formulations. The sodium hexametaphosphate used in the CPC was supplied by Aldrich. X-ray powder diffraction (XRD) data showed three major crystalline phases in $\mathrm{FAF}$ - quartz $\left(\mathrm{SiO}_{2}\right)$, mullite $\left(3 \mathrm{Al}_{2} \mathrm{O}_{3} \cdot 2 \mathrm{SiO}_{2}\right)$ and hematite $\left(\mathrm{Fe}_{2} \mathrm{O}_{3}\right)$. The CAC\#80 encompassed crystalline corundum $\left(\alpha-\mathrm{Al}_{2} \mathrm{O}_{3}\right)$, calcium monoaluminate $\left(\mathrm{CaO} . \mathrm{Al}_{2} \mathrm{O}_{3}\right)$ and calcium dialuminate $\left(\mathrm{CaO} .2 \mathrm{Al}_{2} \mathrm{O}_{3}\right)$, while $\mathrm{CAC \# 51}$ had calcium monoaluminate as the major phase and gehlenite $\left[\mathrm{Ca}_{2} \mathrm{Al}(\mathrm{Al}, \mathrm{Si})_{2} \mathrm{O}_{7}\right]$ and corundum as secondary phases.

The slurries were mixed by hand at a water to blend ratio of 0.4 for the class G/silica blend and at 0.44 for all the other blends. After mixing, the slurries were poured into $20 \times 40 \mathrm{~mm}$ cylindrical moulds and left to set under $100 \%$ humidity at $85^{\circ} \mathrm{C}$ for $24 \mathrm{~h}$, imitating placement temperatures in a geothermal well. Set samples were further cured for $24 \mathrm{~h}$ at $300^{\circ} \mathrm{C}$ in autoclaves, representing early curing under static conditions of high-temperature geothermal wells. The volumetric

\begin{tabular}{llll}
\hline & Cement: wt \% & Class F fly ash (FAF): wt \% & Other blend-forming reactants: wt $\%$ \\
\hline TSRC-1 & $56 \cdot 6$ (CAC\#80) & $37 \cdot 7$ & $5 \cdot 7$ (sodium metasilicate) \\
TSRC-2 & $56 \cdot 6$ (CAC\#51) & $37 \cdot 7$ & $5 \cdot 7$ (sodium metasilicate) \\
TSRC-3 & $37 \cdot 7$ (CAC\#80) & $56 \cdot 6$ & $5 \cdot 7$ (sodium metasilicate) \\
TSRC-4 & $37 \cdot 7$ (CAC\#51) & $56 \cdot 6$ & $5 \cdot 7$ (sodium metasilicate) \\
Class G & $70 \cdot 0$ & - & 30 (silica) \\
CPC & $47 \cdot 2$ (CAC\#51) & $47 \cdot 2$ & $5 \cdot 6$ (sodium hexametaphosphate)
\end{tabular}

Table 1. Mix proportions of cement composites

\begin{tabular}{|c|c|c|c|c|c|c|c|c|}
\hline & $\begin{array}{c}\text { Aluminium } \\
\text { oxide } \\
\left(\mathrm{Al}_{2} \mathrm{O}_{3}\right)\end{array}$ & $\begin{array}{c}\text { Calcium } \\
\text { oxide } \\
(\mathrm{CaO})\end{array}$ & $\begin{array}{c}\text { Silicon } \\
\text { dioxide } \\
\left(\mathrm{SiO}_{2}\right)\end{array}$ & $\begin{array}{c}\text { Iron } \\
\text { oxide } \\
\left(\mathrm{Fe}_{2} \mathrm{O}_{3}\right)\end{array}$ & $\begin{array}{c}\text { Sodium } \\
\text { oxide } \\
\left(\mathrm{Na}_{2} \mathrm{O}\right)\end{array}$ & $\begin{array}{c}\text { Potassium } \\
\text { oxide } \\
\left(\mathrm{K}_{2} \mathrm{O}\right)\end{array}$ & $\begin{array}{c}\text { Titanium } \\
\text { dioxide } \\
\left(\mathrm{TiO}_{2}\right)\end{array}$ & $\begin{array}{c}\text { Sulfur } \\
\text { trioxide } \\
\left(\mathrm{SO}_{3}\right)\end{array}$ \\
\hline Class G cement: atomic \% & $1 \cdot 0$ & 71 & 17 & $5 \cdot 0$ & $0 \cdot 34$ & $1 \cdot 2$ & - & $3 \cdot 6$ \\
\hline CAC\#80: atomic \% & $75 \cdot 2$ & $24 \cdot 7$ & - & $0 \cdot 1$ & - & - & - & - \\
\hline CAC\#51: atomic \% & $45 \cdot 1$ & $49 \cdot 7$ & - & $2 \cdot 8$ & - & - & $2 \cdot 4$ & - \\
\hline FAF: atomic $\%$ & 35 & $2 \cdot 7$ & 50 & $7 \cdot 1$ & $0 \cdot 30$ & $3 \cdot 1$ & $1 \cdot 6$ & - \\
\hline Sodium metasilicate: wt\% & - & - & $46 \cdot 6$ & - & $50 \cdot 5$ & - & - & - \\
\hline
\end{tabular}

Table 2. Oxide compositions of blend-forming components 
proportion of cement to water was 1 to $3 \cdot 5$; the pressure in the autoclave was $8 \cdot 27 \mathrm{MPa}$. Three cured samples of each type were weighed, measured and placed into solutions of sulfuric acid at $90^{\circ} \mathrm{C}(\mathrm{pH} 2.5)$ for $6 \mathrm{~d}$. Although the reactions' kinetic depends on the temperature, technical limitations did not allow acid experiments to be performed at geothermal temperatures of $300^{\circ} \mathrm{C}$. After this treatment the samples were visually examined for signs of erosion and spallation. Since the TSRC samples did not show any visual degradation they were immersed into the acid at $\mathrm{pH} 0.5$ for a further $12 \mathrm{~d}$. The volume of the acid solution was twice the volume of the sample and the solutions were replaced with fresh ones every $3 \mathrm{~d}$ to maintain $<15 \%$ increase in $\mathrm{pH}$ during the treatment.

After acid exposure the specimens were rinsed with water, weighed, measured and tested for compressive strength. They were then ground into very fine powder and dried at $90^{\circ} \mathrm{C}$ for $24 \mathrm{~h}$ before conducting thermogravimetric analyses (TGA), attenuated total reflectance-Fourier transform infrared spectroscopy (ATR-FTIR), $\mu$ EDX measurements and XRD characterisation. The samples were examined using a $40 \mathrm{kV}$, $40 \mathrm{~mA}$ copper anode x-ray tube. The results were analysed using the International Centre for Diffraction Data (ICDD) PDF-4/Minerals 2014 database. The morphologies of the

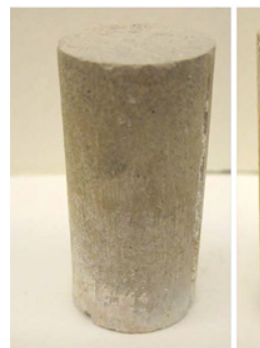

(a)

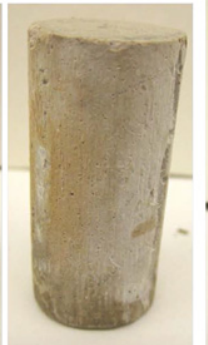

(b)

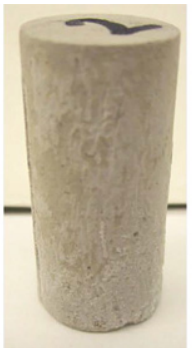

(c)

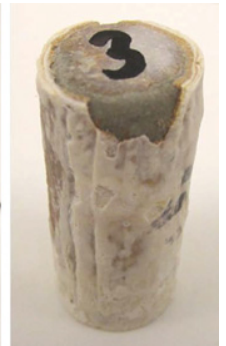

(d)
Figure 1. Appearance of samples after $18 \mathrm{~d}$ exposure to sulfuric acid at $90^{\circ} \mathrm{C}(\mathrm{pH} 2.5$ for $6 \mathrm{~d}$ followed by $\mathrm{pH} 0.5$ for $12 \mathrm{~d}$ ): (a) TSRC-1; (b) TSRC-2; (c) CPC; (d) class G/silica selected formulations were explored on typical spots of freshly fractured and chromium-coated samples with a JEOL $7600 \mathrm{~F}$ scanning electron microscope (SEM). The goal of the study was to obtain information on the TSRC-sulfuric acid reaction products and phase transformations of the original hydrates formed at early ages. These reaction products could either inhibit or accelerate the acid-induced erosion and spallation of geothermal cements.

\section{Results and discussion}

Appearance and mechanical properties

As shown in Figure 1, the TSRC-1, TSRC-2 and CPC samples exposed to acid resembled the control samples; there were some brownish areas on the TSRC-2 and TSRC-4 specimens but the rim structure was not clearly visible; the class G/silica samples formed a well-defined white layer that partially eroded the densified underlying zone.

Table 3 summarises the mechanical properties of the samples before and after acid exposure. The decreases in weight and diameter may result from sample dissolution, erosion and spallation, while the precipitation of acid-cement reaction products may increase these parameters. The processes of weight and diameter gain or loss may partially or completely compensate each other if they happen simultaneously. All the TSRC samples experienced some weight loss, shrank in diameter and had a small corrosion depth, suggesting that product accumulation was less important than cement dissolution in the acid. The CAC-rich samples lost the least weight (TSRC-1) and diameter, possibly by forming expansive calcium salts (TSRC2). An increase in FAF to $60 \mathrm{wt} \%$ (TSRC-3 and TSRC-4) caused further weight and diameter losses.

The compressive strength of the TSRC samples was not compromised by the acid exposure: the strength of TSRC-1 remained unchanged, while the strengths of TSRC-2, TSRC-3 and TSRC-4 increased by $17 \%, 31 \%$ and $67 \%$, respectively. Continuous hydration and FAF reactions that are slower than

Before acid exposure After acid exposure

\begin{tabular}{|c|c|c|c|c|c|}
\hline TSRC-1 & $-0 \cdot 3$ & -0.79 & $18 \cdot 0 \pm 0 \cdot 7$ & $18 \cdot 0 \pm 2 \cdot 0$ & 0.68 \\
\hline TSRC-2 & $-1 \cdot 12$ & -0.02 & $13 \cdot 9 \pm 1 \cdot 3$ & $16 \cdot 3 \pm 1 \cdot 7$ & 0.44 \\
\hline TSRC-3 & $-1 \cdot 79$ & $-1 \cdot 65$ & $11 \cdot 2 \pm 1 \cdot 9$ & $14 \cdot 7 \pm 2 \cdot 3$ & $<0 \cdot 5$ \\
\hline TSRC-4 & $-3 \cdot 17$ & -0.85 & $12 \cdot 7 \pm 2 \cdot 1$ & $21 \cdot 2 \pm 4 \cdot 5$ & $<0.5$ \\
\hline Class G/silica & $+3 \cdot 83$ & $+1 \cdot 88$ & $17 \cdot 4 \pm 0.7$ & $20 \cdot 5 \pm 0.6$ & 1.97 \\
\hline CPC & $-3 \cdot 20$ & $-1 \cdot 7$ & $25 \cdot 3 \pm 1 \cdot 6$ & $24 \cdot 6 \pm 6 \cdot 5$ & 0.86 \\
\hline
\end{tabular}

Table 3. Sample properties after $18 \mathrm{~d}$ exposure to sulfuric acid at $90^{\circ} \mathrm{C}(\mathrm{pH} 2.5$ for $6 \mathrm{~d}$ followed by $\mathrm{pH} 0.5$ for $12 \mathrm{~d}$ ) 

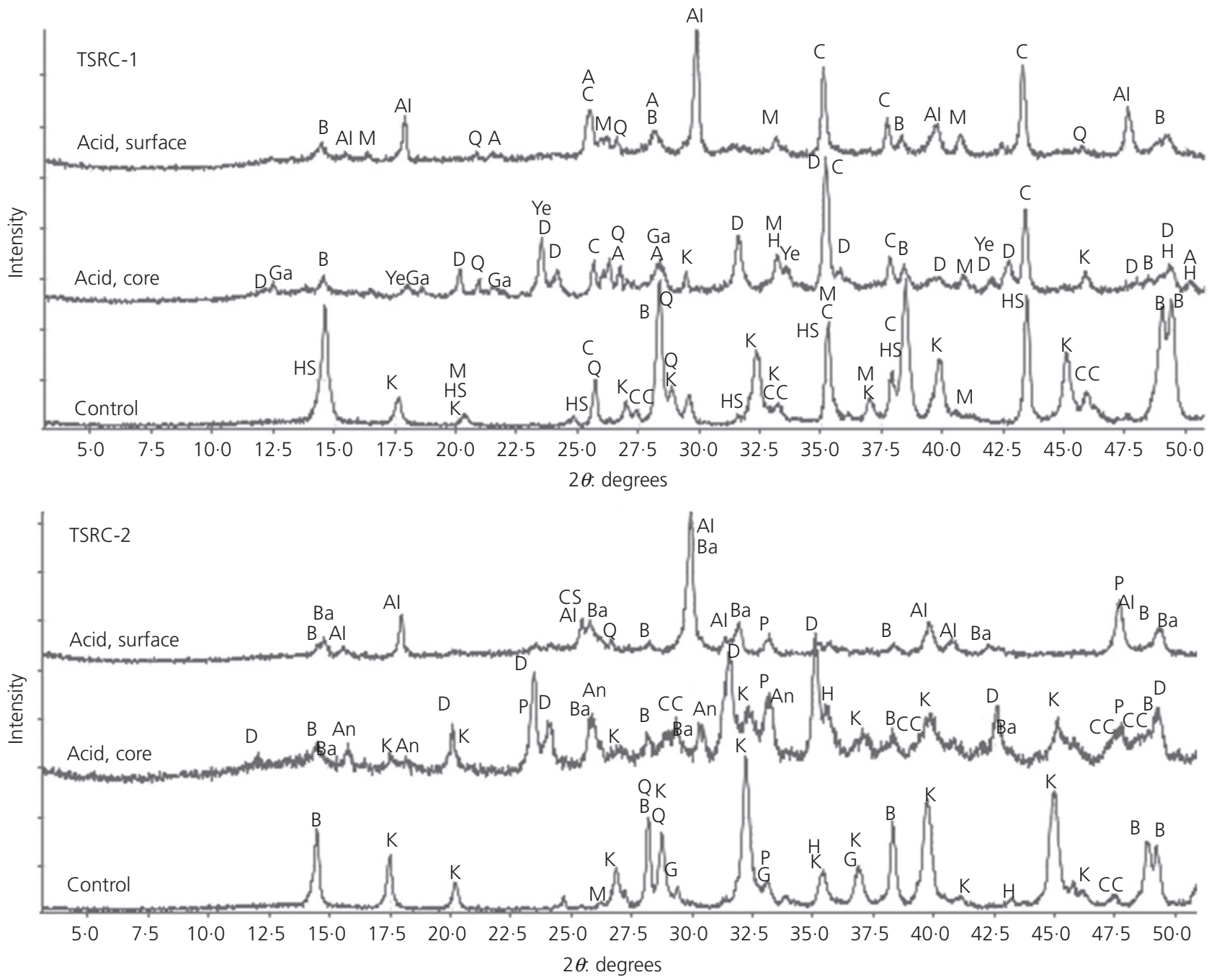

Figure 2. XRD patterns of TSRC-1 and TSRC-2 samples exposed to sulfuric acid for $18 \mathrm{~d}$ compared with control samples before exposure: B, boehmite; K, katoite; Q, quartz; G, gehlenite;

$\mathrm{P}$, perovskite; $\mathrm{H}$, hematite; $\mathrm{CC}$, calcium carbonates; An, analcime;
Ba, bassanite; CS, anhydrate; Al, alunite; D, dmisteinbergite; Ye, ye'elimite; $M$, mullite; $H S$, hydroxysodalite; Ga, garronite; A, anorthite; C, corundum the hydration of CAC could be responsible for the increased compressive strength, especially in the FAF-rich samples (TSRC-3 and TSRC-4).

The CPC sample had the largest losses in weight and diameter, and experienced a $3 \%$ decrease in compressive strength. The class $\mathrm{G} /$ silica sample increased in both weight and diameter by forming a large external layer that proved to be calcium sulfate in the XRD tests; the compressive strength of this sample increased by about $19 \%$.

\section{Analyses of degraded samples - crystalline phases,} XRD patterns

Figures 2-4 show the XRD patterns of the tested cement formulations cured at $300^{\circ} \mathrm{C}$ before and after acid exposure.
Table 4 lists the crystalline phases identified in the samples, along with their ICDD numbers and chemical formulas. Tables 5-7 give more detailed information on $2 \theta$ values of the experimental peaks and the phases associated with them. The complex composites show patterns with split peaks, suggesting the contribution of several phases, so each peak on the XRD patterns is associated with a number of possible contributors in the tables.

Crystalline phases of the control TSRC samples included nonhydrated reactants (corundum, quartz, mullite, perovskite, gehlenite, hematite and some calcium carbonates), hydration products of CAC such as boehmite and hydrogrossular minerals (katoite silician and katoite), and hydration and reaction products from FAF alone or from both FAF and CAC 

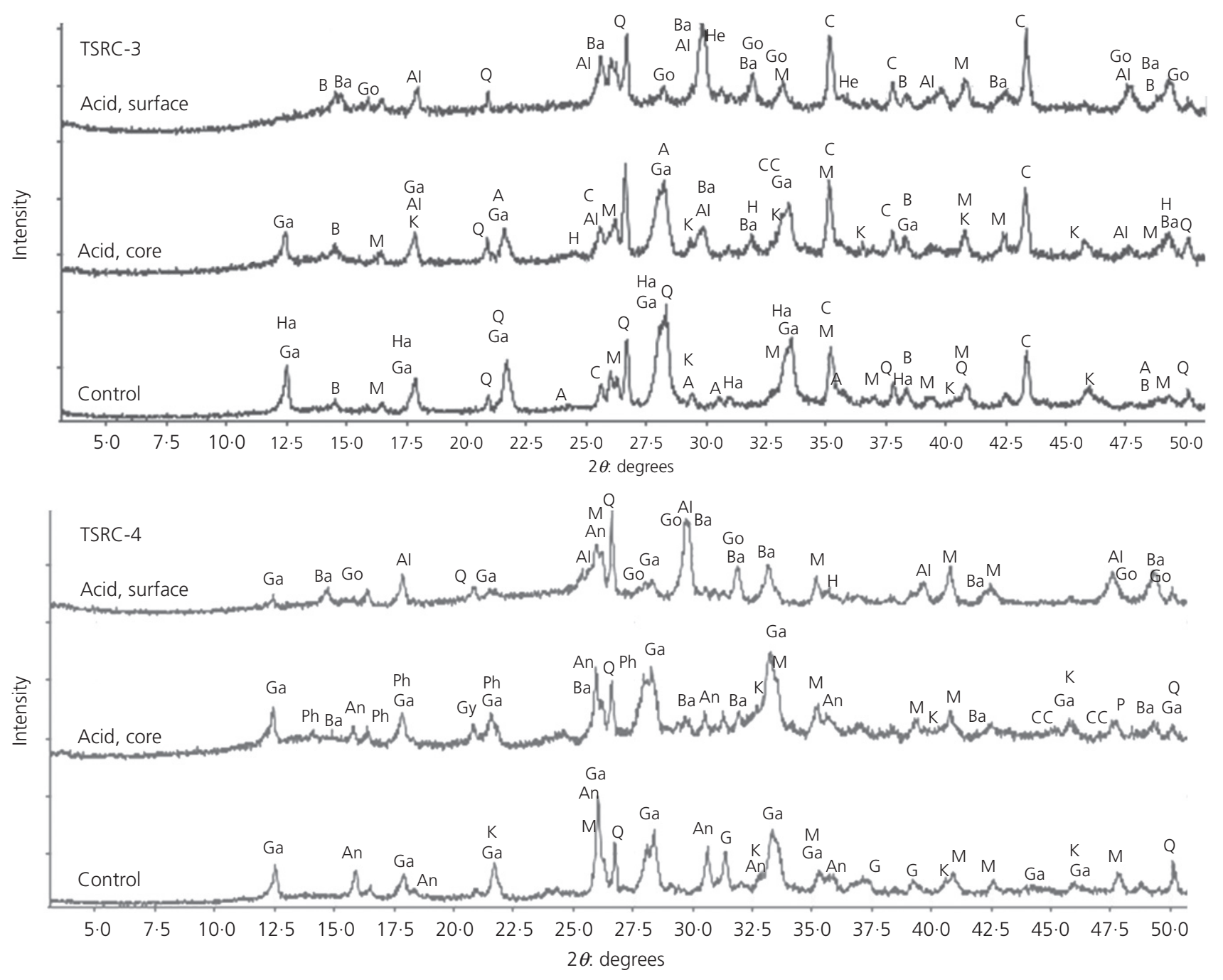

Figure 3. XRD patterns of TSRC-3 and TSRC-4 samples exposed to sulfuric acid for $18 \mathrm{~d}$ compared with control samples before exposure: Ga, garronite; Go, gorgeyite; B, boehmite; K, katoite; $\mathrm{Q}$, xquartz; $\mathrm{G}$, gehlenite; $\mathrm{H}$, hematite; $\mathrm{CC}$, calcium carbonates;
An, analcime; $\mathrm{Ba}$, bassanite; Al, alunite; $\mathrm{D}$, dmisteinbergite; $\mathrm{M}$, mullite; $\mathrm{Ha}$, harmotome; $\mathrm{C}$, corundum; $\mathrm{Ph}$, phillipsite; He, hedenbergite (such as various zeolites and anorthite). (Other members of the garnet series, such as grossular and hibschite, or the plagioclase feldspar series ranging from albite to anorthite may contribute to the XRD patterns but were not explicitly identified because of the blends' complexity and their overlapping XRD patterns.) In addition, calcium carbonates formed during sample mixing and curing since no special precautions were taken to avoid carbonation.

CAC hydrates (boehmite, katoite silician and katoite) crystallised as major phases in the cement-rich blends TSRC-1 and TSRC-2. In the higher calcium content blend (TSRC-2), katoite formed instead of katoite silician, and the intensity of boehmite peaks was significantly lower than in the patterns of the aluminium- rich blend (TSRC-1). TSRC-1 hydrates included not only the faster hydrating CAC hydration products, but also a crystalline zeolite, hydroxysodalite, which is a product of fly ash reactions.

An increase in FAF content in the initial blends (TSRC-3 and TSRC-4) raised the intensities of the fly ash hydration products, zeolites, and weakened those of the CAC hydrates, hydrogrossular phases and boehmite.

During the $18 \mathrm{~d}$ acid treatment, curing of the samples continued but at a lower temperature of $90^{\circ} \mathrm{C}$, causing changes mostly in compositions of the sample cores, and the acid reacted with the cement, mostly altering the surface layers. The following changes occurred in the cores of TSRC samples. 

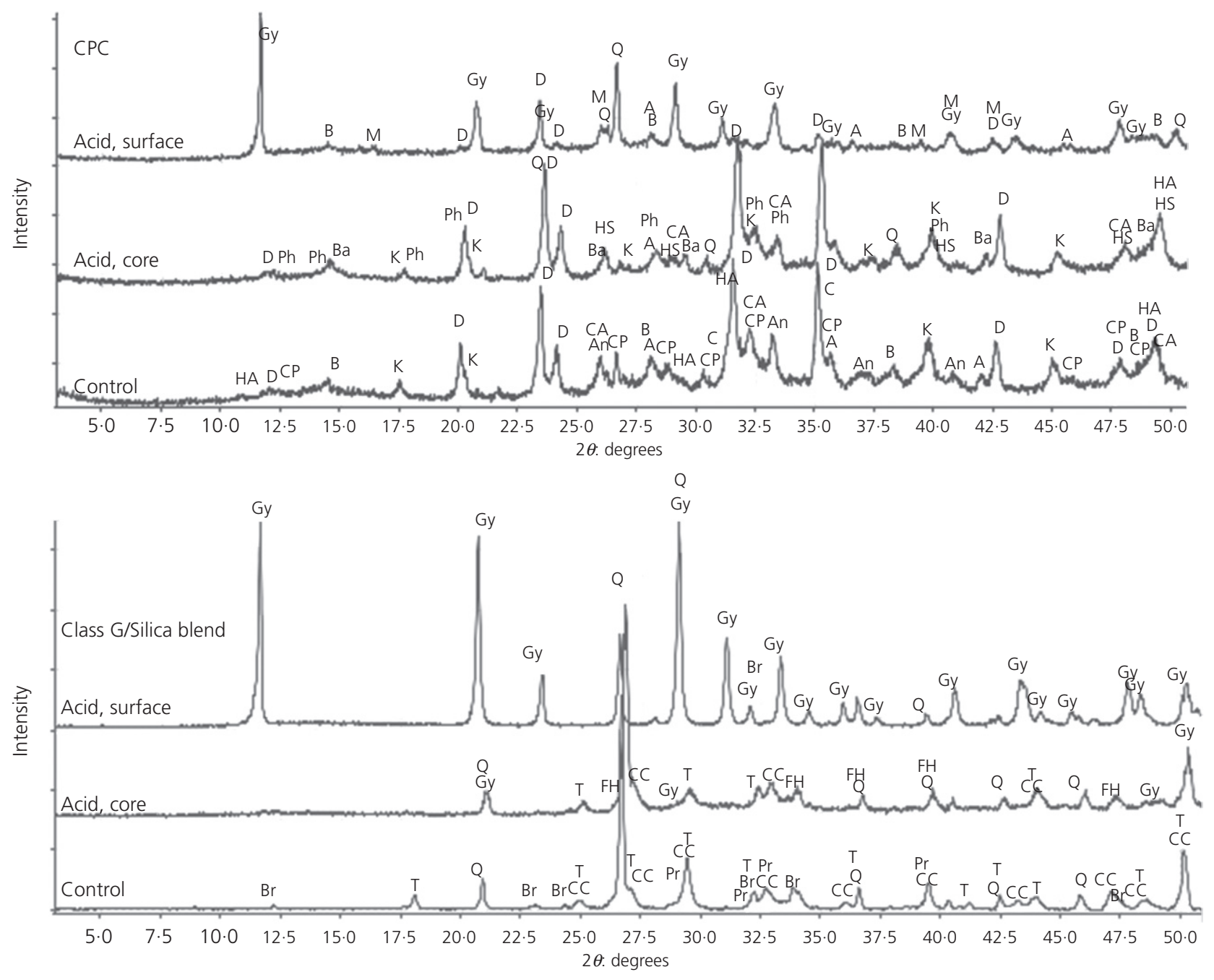

Figure 4. XRD patterns of CPC and class G/silica cement samples exposed to sulfuric acid for $18 \mathrm{~d}$ compared with control samples before exposure: $T$, tobermorite $9 \mathrm{~A} ; \mathrm{Q}$, quartz; $C \mathrm{C}$, calcium carbonate; $\mathrm{Br}$, brownmillerite; $\mathrm{Pr}$, portlandite; $\mathrm{FH}$, iron oxide hydroxide; Gy, gypsum; D, dmisteinbergite; K, katoite;
An, analcime; $C P$, calcium hydrogen phosphate; $A$, anorthite; CA, carbonated hydroxylapatite; HA, hydroxylapatite; $\mathrm{HS}$, hydroxylapatite sulfatian; $\mathrm{Ph}$, phillipsite; $\mathrm{Ba}$, bassanite; $M$, mullite; $C$, corundum
For CAC-rich blends TSRC-1 and TSRC-2, the feldspar family minerals, isomorphic dmisteinbergite and anorthite, replaced the garnet series hydrates, katoites; the intensities of boehmite peaks dropped, although it remained one of the major phases. Further reactions of FAF produced new zeolites such as analcime, garronite and harmotome (and, possibly, other zeolites with chains of double-connected fourmembered rings, gismondine and phillipsite, which have overlapping XRD patterns). In addition, some calcium sulfates were identifiable in the core of TSRC-2, but not to any significant extent in the core of TSRC-1 (Table 4).

The cores of the FAF-rich TSRC-3 and TSRC-4 samples showed partial or complete disappearance of the originally formed zeolites. In both TSRC-3 and TSRC-4, sulfur permeated into the cores of the samples with the formation of calcium sulfates in TSRC-4 (made with calcium-rich CAC\#51) and potassium-aluminium sulfate in TSRC-3 (made with aluminium-rich CAC\#80). Calcium carbonates persisted in the cores of all the samples but not in the acid-exposed surfaces.

In the surfaces of the acid-exposed samples, calcium sulfates and potassium-aluminium sulfate phases partially or completely replaced the peaks of most calcium-containing hydrates, including hydrogrossular, plagioclase feldspar and zeolites. Exceptionally, there were no crystalline calcium sulfates in the surface layer of TSRC-1. Only potassium(sodium) aluminium sulfate, alunite, formed in the surface of this sample. In geothermal sites, alunite forms naturally as a result of 


\section{TSRC-1 before exposure}

Boehmite $\mathrm{AlO}(\mathrm{OH})$ (01-074-2895)

Hydroxysodalite $\mathrm{Na}_{4} \mathrm{Al}_{3} \mathrm{Si}_{3} \mathrm{O}_{12}(\mathrm{OH})(00-011-0401)$

Katoite $\mathrm{Ca}_{3} \mathrm{Al}_{2}\left(\mathrm{SiO}_{4}\right)(\mathrm{OH})_{8}(04-014-1869)$

TSRC-1 after exposure, core

Dmisteinbergite $\mathrm{CaAl}_{2} \mathrm{Si}_{2} \mathrm{O}_{8}(04-011-5220)$

Boehmite $\mathrm{AlO}(\mathrm{OH})$ (01-074-2896)

TSRC-1 after exposure, surface

Alunite $\mathrm{KAl}_{3}\left(\mathrm{SO}_{4}\right)(\mathrm{OH})_{6}(00-047-1884)$

Boehmite AlOOH (01-073-9093)

TSRC-2 before exposure

Boehmite $\mathrm{AlO}(\mathrm{OH})(04-010-5683)$

Katoite $\mathrm{Ca}_{3} \mathrm{Al}_{2}(\mathrm{OH})_{12}$ (04-017-4319)

TSRC-2 after exposure, core

Dmistenbeirgite $\mathrm{CaAl}_{2} \mathrm{Si}_{2} \mathrm{O}_{8}(00-051-0064)$

Bassanite $\mathrm{CaSO}_{4}\left(\mathrm{H}_{2} \mathrm{O}\right)_{0.67}(00-036-0617)$

Boehmite $\mathrm{AlO}(\mathrm{OH})(04-010-5683)$

TSRC-2 after exposure, surface

Alunite $\left(\mathrm{K}_{0.805} \mathrm{Na}_{0.132}\left(\mathrm{H}_{2} \mathrm{O}\right)_{0.063}\right) \mathrm{Al}_{3}\left(\mathrm{SO}_{4}\right)_{2}(\mathrm{OH})_{6}$

(01-075-9141/00-001-0879)

Boehmite $\mathrm{AlO}(\mathrm{OH})(04-010-5683)$

Bassanite $\mathrm{Ca}_{3}\left(\mathrm{SO}_{4}\right)_{3}\left(\mathrm{H}_{2} \mathrm{O}\right)_{1 \cdot 8}(04-011-1767 / 01-072-4535)$

TSRC-3 before exposure

Anorthite $\mathrm{CaAl}_{2} \mathrm{Si}_{2} \mathrm{O}_{8}(00-012-0301 / 04-011-2514)$

Garronite $\mathrm{Na}_{8} \mathrm{Ca}_{2 \cdot 82}\left(\mathrm{Al}_{6} \mathrm{Si}_{10} \mathrm{O}_{32}\right)\left(\mathrm{H}_{2} \mathrm{O}\right)_{12.08} /$

$\mathrm{Na}_{0.1} \mathrm{Ca}_{1.35} \mathrm{Al}_{2.8} \mathrm{Si}_{5 \cdot 2} \mathrm{O}_{16}\left(\mathrm{H}_{2} \mathrm{O}\right)_{7}(01-079-1336 / 04-0121546)$

Harmotome $\mathrm{Na}_{3} \mathrm{Al}_{3} \mathrm{Si}_{5} \mathrm{O}_{16}\left(\mathrm{H}_{2} \mathrm{O}\right)_{6 \cdot 5}$ (04-009-5432)
Calcium carbonate $\mathrm{CaCO}_{3}$ (04-006-5444/01-085-0849)

Quartz $\mathrm{SiO}_{2}(04-006-2057)$

Corundum $\mathrm{Al}_{2} \mathrm{O}_{3}$ (04-008-3293)

Mullite $\mathrm{Al}_{6} \mathrm{Si}_{12} \mathrm{O}_{13}(00-015-0776)$

Hematite $\mathrm{Fe}_{2} \mathrm{O}_{3}$ (00-001-1053)

Anorthite $\mathrm{CaAl}_{2} \mathrm{Si}_{2} \mathrm{O}_{8}$ (04-012-1276)

Garronite $\mathrm{Ca}_{3} \mathrm{Al}_{2}\left(\mathrm{SiO}_{4}\right)_{3}(00-039-1374)$

Calcium carbonate $\mathrm{CaCO}_{3}$ (04-006-5444/01-085-0849)

Harmotome $\mathrm{Na}_{3} \mathrm{Al}_{3} \mathrm{Si}_{5} \mathrm{O}_{16}\left(\mathrm{H}_{2} \mathrm{O}\right)_{6.5}$ (04-009-5432)

Analcime $\mathrm{Na}_{8} \mathrm{Al}_{8} \mathrm{Si}_{16} \mathrm{O}_{48}\left(\mathrm{H}_{2} \mathrm{O}\right)_{8}(04-011-6233)$

Hydroxysodalite $\mathrm{Na}_{4} \mathrm{Al}_{3} \mathrm{Si}_{3} \mathrm{O}_{12}(\mathrm{OH})(00-011-0401)$

Katoite $\mathrm{Ca}_{3} \mathrm{Al}_{2}\left(\mathrm{SiO}_{4}\right)(\mathrm{OH})_{8}(04-014-1869)$

Anorthite $\mathrm{CaAl}_{2} \mathrm{Si}_{2} \mathrm{O}_{8}(00-041-1486)$

Analcime $\mathrm{Na}_{8} \mathrm{Al}_{8} \mathrm{Si}_{16} \mathrm{O}_{48}\left(\mathrm{H}_{2} \mathrm{O}\right)_{8}(04-011-7963)$

Calcite $\mathrm{Ca}\left(\mathrm{CO}_{3}\right)(01-083-0577)$

Katoite $\mathrm{Ca}_{2.93} \mathrm{Al}_{1.97}\left(\mathrm{Si}_{0.64} \mathrm{O}_{2.56}\right)(\mathrm{OH})_{9.44}(01-077-1713)$

Analcime $\mathrm{Na}_{1 \cdot 71}\left(\left(\mathrm{Al}_{1 \cdot 806} \mathrm{Si}_{4 \cdot 194}\right) \mathrm{O}_{12}\right)\left(\mathrm{H}_{2} \mathrm{O}\right)_{2 \cdot 16}(01-075-8689)$ Anhydrate $\mathrm{CaSO}_{4}(01-072-0916)$

Gypsum $\mathrm{CaSO}_{4}\left(\mathrm{H}_{2} \mathrm{O}\right)_{2}$ (01-079-5985)

Calcite $\mathrm{CaCO}_{3}(01-080-2791 / 01-083-0577)$

Dmisteinbergite $\mathrm{CaAl}_{2} \mathrm{Si}_{2} \mathrm{O}_{8}$ (00-051-0064)

Anhydrate $\mathrm{CaSO}_{4}(01-072-0916)$

Boehmite AlOOH (00-021-1307)

Gobbinsite $\mathrm{Na}_{3} \mathrm{Al}_{3} \mathrm{Si}_{5} \mathrm{O}_{66}\left(\mathrm{H}_{2} \mathrm{O}\right)_{6}(00-025-0779)$

Katoite $\mathrm{Ca}_{3} \mathrm{Al}_{2}\left(\mathrm{SiO}_{4}\right)_{1.53}(\mathrm{OH})_{5 \cdot 88}(04-014-9841)$

Analcime $\mathrm{Na}_{8} \mathrm{Al}_{8} \mathrm{Si}_{16} \mathrm{O}_{48}\left(\mathrm{H}_{2} \mathrm{O}\right)_{8}(04-011-7963)$

Gismondine (01-076-8378)

Calcium carbonate $\mathrm{CaCO}_{3}$ (04-017-9180/04-002-9082)
Corundum $\mathrm{Al}_{2} \mathrm{O}_{3}$ (04-005-4497)

Mullite $\mathrm{Al}_{6} \mathrm{Si}_{12} \mathrm{O}_{13}(00-015-0776)$

Quartz $\mathrm{SiO}_{2}(01-077-8621)$

Hematite $\mathrm{Fe}_{2} \mathrm{O}_{3}(01-080-5407 / 08 / 09 /$ 04-002-5211)

Corundum $\mathrm{Al}_{2} \mathrm{O}_{3}$ (04-005-4497) Mullite $\mathrm{Al}_{6} \mathrm{Si}_{12} \mathrm{O}_{13}(01-079-1452)$

Quartz $\mathrm{SiO}_{2}(01-083-0539)$

Hematite $\mathrm{Fe}_{2} \mathrm{O}_{3}$ (04-002-2983)

Gehlenite $\mathrm{Al}_{2} \mathrm{Ca}_{2} \mathrm{O}_{7} \mathrm{Si}$ (04-015-7930)

Quartz $\mathrm{SiO}_{2}$ (01-083-0542/01-073-1748)

Perovskite $\mathrm{CaTiO}_{3}(00-022-0153)$

Hematite $\mathrm{Fe}_{2} \mathrm{O}_{3}(00-039-1346)$

Hematite $\mathrm{Fe}_{2} \mathrm{O}_{3}$ (04-014-7214)

Gehlenite $\mathrm{Al}_{2} \mathrm{Ca}_{2} \mathrm{O}_{7} \mathrm{Si}$ (04-015-7930)

Perovskite $\mathrm{CaTiO}_{3}$ (01-081-0561)

Quartz $\mathrm{SiO}_{2}$ (04-007-1438/04-011-9932/01-0751555)

Perovskite $\mathrm{CaTiO}_{3}(00-022-0153)$

Hematite $\mathrm{Fe}_{2} \mathrm{O}_{3}(00-001-1053)$

Corundum $\mathrm{Al}_{2} \mathrm{O}_{3}(04-013-1687 / 04-005-4500)$ Quartz $\mathrm{SiO}_{2}$ (01-085-0794/01-086-1564) Mullite $\left(\mathrm{Al}_{2.34} \mathrm{Si}_{0.66}\right) \mathrm{O}_{4.83}(01-076-2579)$ 
TSRC-3 after exposure, core

Katoite silician $\mathrm{Ca}_{3} \mathrm{Al}_{2}\left(\mathrm{SiO}_{4}\right)_{1.53}(\mathrm{OH})_{5.88}(04-014-9841)$

TSRC-3 after exposure, surface

Boehmite AlOOH (04-010-5683/04016-2858)

Bassanite $\mathrm{CaSO}_{4}\left(\mathrm{H}_{2} \mathrm{O}\right)_{0.67}(00-036-0617)$

\section{TSRC-4 before exposure}

Analcime $\mathrm{Na}_{8} \mathrm{Al}_{8} \mathrm{Si}_{16} \mathrm{O}_{48}\left(\mathrm{H}_{2} \mathrm{O}\right)_{8}(04-011-7963)$

Garronite $\mathrm{NaCa}_{2 \cdot 5}\left(\mathrm{Al}_{6} \mathrm{Si}_{10}\right) \mathrm{O}_{32} \cdot 14 \mathrm{H}_{2} \mathrm{O}(00-059-0383)$

TSRC-4 after exposure, core

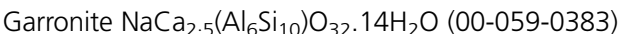

\section{TSRC-4 after exposure, surface}

Alunite $\mathrm{KAl}_{3}\left(\mathrm{SO}_{4}\right)_{2}(\mathrm{OH})_{6}(00-047-1885)$

Bassanite $\mathrm{Ca}_{0.98} \mathrm{Na}_{0.03} \mathrm{SO}_{4} \cdot 0 \cdot 47 \mathrm{H}_{2} \mathrm{O}(00-037-0246)$
Boehmite AlOOH (01-074-2897/01-074-2896)

Garronite $\mathrm{NaCa}_{2 \cdot 5}\left(\mathrm{Al}_{6} \mathrm{Si}_{10}\right) \mathrm{O}_{32}\left(\mathrm{H}_{2} \mathrm{O}\right)_{14}(00-059-0383)$

Anorthite $\mathrm{CaAl}_{2} \mathrm{Si}_{2} \mathrm{O}_{8}$ (04-011-2883)

Alunite $\mathrm{KAl}_{3}\left(\mathrm{SO}_{4}\right)(\mathrm{OH})_{6}(00-047-1884)$

Katoite $\mathrm{Ca}_{3} \mathrm{Al}_{2}(\mathrm{OH})_{12}(04-017-4320)$

Analcime $\mathrm{Na}_{8} \mathrm{Al}_{8} \mathrm{Si}_{16} \mathrm{O}_{48}\left(\mathrm{H}_{2} \mathrm{O}\right)_{8}(04-011-6756)$

Gismondine (01-076-8378)

Calcium carbonate $\mathrm{CaCO}_{3}$ (04-017-9180/04-002-9082)

Alunite $\mathrm{KAl}_{3}\left(\mathrm{SO}_{4}\right)(\mathrm{OH})_{6}(04-014-9927)$

Gorgeyite $\mathrm{K}_{2} \mathrm{Ca}_{5}\left(\mathrm{SO}_{4}\right)_{6}\left(\mathrm{H}_{2} \mathrm{O}\right)(00-018-0997)$

Analcime $\mathrm{Na}_{8} \mathrm{Al}_{8} \mathrm{Si}_{16} \mathrm{O}_{48}\left(\mathrm{H}_{2} \mathrm{O}\right)_{8}(04-011-6233)$

Anhydrate $\mathrm{CaSO}_{4}$ (04-007-4744)

Hedenbergite $\mathrm{Na}_{0.45} \mathrm{Ca}_{0.55} \mathrm{FeSi}_{2} \mathrm{O}_{6}$ (04-013-1878)

Phillipsite $\mathrm{K}(\mathrm{K}, \mathrm{Na})_{2}(\mathrm{Si}, \mathrm{Al})_{8} \mathrm{O}_{16} .4 \mathrm{H}_{2} \mathrm{O}(00-046-1427)$ Gismondine $\mathrm{Ca}_{4}\left(\mathrm{Al}_{8} \mathrm{Si}_{8} \mathrm{O}_{32}\right)\left(\mathrm{H}_{2} \mathrm{O}\right)_{19}$ (01-076-8378)

Katoite $\mathrm{Ca}_{3} \mathrm{Al}_{2}(\mathrm{OH})_{12}(04-017-4322)$

Calcium carbonate $\mathrm{CaCO}_{3}$ (04-014-1837/01-081-9561)

Phillipsite $(\mathrm{K}, \mathrm{Na})_{2}(\mathrm{Si}, \mathrm{Al})_{8} \mathrm{O}_{16} .4 \mathrm{H}_{2} \mathrm{O}(00-046-1427)$ Bassanite $\mathrm{Ca}_{0.98} \mathrm{Na}_{0.03} \mathrm{SO}_{4} .0 \cdot 47 \mathrm{H}_{2} \mathrm{O}(00-037-0246)$ Analcime $\mathrm{Na}_{8} \mathrm{Al}_{8} \mathrm{Si}_{16} \mathrm{O}_{48}\left(\mathrm{H}_{2} \mathrm{O}\right)_{8}(04-011-7963)$

Gismondine $\mathrm{Ca}_{4}\left(\mathrm{Al}_{8} \mathrm{Si}_{8} \mathrm{O}_{32}\right)\left(\mathrm{H}_{2} \mathrm{O}\right)_{19}(01-076-8378)$

Gypsum $\mathrm{CaSO}_{4} 2 \mathrm{H}_{2} \mathrm{O}$ (04-010-9409)

Katoite $\mathrm{Ca}_{3} \mathrm{Al}_{2}(\mathrm{OH})_{12}(04-017-4322$

Calcium carbonate $\mathrm{CaCO}_{3}$ (04-014-1837/01-081-9561)

Gorgeyite $\mathrm{K}_{2} \mathrm{Ca}_{5}\left(\mathrm{SO}_{4}\right)_{6}\left(\mathrm{H}_{2} \mathrm{O}\right)(04-012-5273)$

Analcime $\mathrm{Na}_{8} \mathrm{Al}_{8} \mathrm{Si}_{16} \mathrm{O}_{48}\left(\mathrm{H}_{2} \mathrm{O}\right)_{8}(04-011-7963)$

Anhydrate $\mathrm{CaSO}_{4}(01-074-2421)$

Phillipsite $(\mathrm{K}, \mathrm{Na})_{2}(\mathrm{Si}, \mathrm{Al})_{8} \mathrm{O}_{16} .4 \mathrm{H}_{2} \mathrm{O}(00-046-1427)$

Garronite $\mathrm{NaCa}_{2.5}\left(\mathrm{Al}_{6} \mathrm{Si}_{10}\right) \mathrm{O}_{32} .14 \mathrm{H}_{2} \mathrm{O}(00-059-0383)$

Gismondine $\mathrm{Ca}_{4}\left(\mathrm{Al}_{8} \mathrm{Si}_{8} \mathrm{O}_{32}\right)\left(\mathrm{H}_{2} \mathrm{O}\right)_{19}(01-076-8378)$
Quartz $\mathrm{SiO}_{2}$ (01-078-1252)

Hematite $\mathrm{Fe}_{2} \mathrm{O}_{3}$ (04-015-9577/01-080-5406)

Corundum $\mathrm{Al}_{2} \mathrm{O}_{3}$ (01-0751862)

Mullite $\mathrm{Al}\left(\mathrm{Al}_{0.69} \mathrm{Si}_{1.22} \mathrm{O}_{4.85}\right)(01-089-2644)$

uartz $\mathrm{SiO}_{2}(04-008-2359)$

Corundum $\mathrm{Al}_{2} \mathrm{O}_{3}$ (04-015-8996)

Mullite $\mathrm{Al}_{2}\left(\mathrm{Al}_{2.8} \mathrm{Si}_{1.2}\right) \mathrm{O}_{9.54}$ (01-084-1205)

Quartz $\mathrm{SiO}_{2}(01-085-0865)$

Gehlenite $\mathrm{Al}_{2} \mathrm{Ca}_{2} \mathrm{O}_{7} \mathrm{Si}$ (04-015-7930)

Mullite $\mathrm{Al}\left(\mathrm{Al}_{0.69} \mathrm{Si}_{1.22} \mathrm{O}_{4.85}\right)(01-089-2644)$

Hematite $\mathrm{Fe}_{2} \mathrm{O}_{3}$ (00-001-1053)

Perovskite $\mathrm{CaTiO}_{3}(00-022-0153$

Quartz $\mathrm{SiO}_{2}$ (01-085-0865)

Gehlenite $\mathrm{Al}_{2} \mathrm{Ca}_{2} \mathrm{O}_{7} \mathrm{Si}$ (04-015-7930)

Perovskite (00-022-0153)

Mullite $\mathrm{Al}\left(\mathrm{Al}_{0.69} \mathrm{Si}_{1.22} \mathrm{O}_{4.85}\right)(01-089-2644)$

Hematite $\mathrm{Fe}_{2} \mathrm{O}_{3}$ (00-001-1053)

Quartz $\mathrm{SiO}_{2}(01-075-8320)$

Gehlenite $\mathrm{Al}_{2} \mathrm{Ca}_{2} \mathrm{O}_{7} \mathrm{Si}$ (04-015-7930)

Mullite $\mathrm{Al}\left(\mathrm{Al}_{0.69} \mathrm{Si}_{1.22} \mathrm{O}_{4.85}\right)(01-089-2644)$

Perovskite $\mathrm{CaTiO}_{3}$ (00-022-0153)

Hematite $\mathrm{Fe}_{2} \mathrm{O}_{3}$ (00-001-1053) 


\section{CPC before exposure}

Dmisteinbergite $\mathrm{CaAl}_{2} \mathrm{Si}_{2} \mathrm{O}_{8}(00-051-0064)$

CPC after exposure, core

Dmisteinbergite $\mathrm{CaAl}_{2} \mathrm{Si}_{2} \mathrm{O}_{8}$ (04-011-6236)

Hydroxylapatite $\mathrm{Ca}_{4.758}\left(\mathrm{H}_{0.21}\left(\mathrm{PO}_{4}\right)\right)(\mathrm{OH})_{0.726}(01-07409764)$

CPC after exposure, surface

Gypsum $\mathrm{CaSO}_{4}\left(\mathrm{H}_{2} \mathrm{O}\right)_{2}$

(04-009-1810/00-033-0311/04-015-4421)

Class $\mathrm{G} / \mathrm{silica}$ before exposure

Tobermorite $9 \mathrm{~A} \mathrm{Ca}_{5} \mathrm{Si}_{6} \mathrm{O}_{16}(\mathrm{OH})_{2}$ (04-012-1761)

Calcium carbonate $\mathrm{CaCO}_{3}$

(04-006-6528/04-011-5985/01-075-9987)

Class $\mathrm{G} /$ silica after exposure, core

Tobermorite $9 \mathrm{~A} \mathrm{Ca}_{5} \mathrm{Si}_{6} \mathrm{O}_{16}(\mathrm{OH})_{2}$ (04-012-1761)

Gypsum $\mathrm{CaSO}_{4}\left(\mathrm{H}_{2} \mathrm{O}\right)_{2}(00-033-0311)$

Class $\mathrm{G} / \mathrm{silica}$ after exposure, surface

Gypsum $\mathrm{CaSO}_{4}\left(\mathrm{H}_{2} \mathrm{O}\right)_{2}(00-033-0311)$

\section{Anorthite $\mathrm{CaAl}_{2} \mathrm{Si}_{2} \mathrm{O}_{8}$ (00-041-1486)}

Calcium hydrogen phosphate $\mathrm{CaHPO}_{4}$ (01-075-1520)

Analcime $\mathrm{NaSi}_{2} \mathrm{AlO}_{6} \mathrm{H}_{2} \mathrm{O}(00-019-1180)$

Carbonated hydroxylapatite $\left(\mathrm{Ca}_{3.13} \mathrm{Na}_{0.53}\right)\left(\mathrm{Ca}_{4.66} \mathrm{Na}_{0.79}\right)$

$\left(\mathrm{PO}_{4}\right)_{4 \cdot 3}\left(\mathrm{CO}_{3}\right)_{1 \cdot 7}(\mathrm{OH})_{0 \cdot 74}\left(\mathrm{H}_{2} \mathrm{O}\right)_{1 \cdot 3}(01-073-7334)$

Katoite silician $\mathrm{Ca}_{3} \mathrm{Al}_{2}\left(\mathrm{SiO}_{4}\right)(\mathrm{OH})_{8}(00-038-0368)$

Portlandite $\mathrm{Ca}(\mathrm{OH})_{2}(00-050-0008)$

Calcium carbonate $\mathrm{CaCO}_{3}(04-014-1601)$

Hydroxylapatite $\mathrm{Ca}_{5}\left(\mathrm{PO}_{4}\right)_{3}(\mathrm{OH})(04-016-1709)$

Boehmite AlOOH (01-074-2896)

Carbonated hydroxylapatite $\left(\mathrm{Ca}_{3.13} \mathrm{Na}_{0.53}\right)\left(\mathrm{Ca}_{4.66} \mathrm{Na}_{0.79}\right)$

$\left(\mathrm{PO}_{4}\right)_{4 \cdot 3}\left(\mathrm{CO}_{3}\right)_{1 \cdot 7}(\mathrm{OH})_{0 \cdot 74}\left(\mathrm{H}_{2} \mathrm{O}\right)_{1 \cdot 3}(01-073-7334)$

Anorthite $\mathrm{CaAl}_{2} \mathrm{Si}_{2} \mathrm{O}_{8}$ (04-012-1276)

Phillipsite $\mathrm{K}_{2 \cdot 5} \mathrm{NaSi}_{11 \cdot 3} \mathrm{Al}_{4 \cdot 7} \mathrm{O}_{32 .} \cdot 13 \mathrm{H}_{2} \mathrm{O}(00-051-1497)$

Katoite $\mathrm{Ca}_{2.93} \mathrm{Al}_{1.97}\left(\mathrm{Si}_{0.64} \mathrm{O}\right)(\mathrm{OH})_{9.44}(01-077-1713)$

Calcium hydrogen phosphate $\mathrm{CaHPO}_{4}$ (01-075-1520)

Calcium carbonate $\mathrm{CaCO}_{3}$ (04-014-1601)

Hydroxylapatite sulfonated $\mathrm{Ca}_{4.95}\left(\mathrm{P} \mathrm{O}_{4}\right)_{2 \cdot 9}\left(\mathrm{SO}_{4}\right)_{0 \cdot 1}(\mathrm{OH})(04-013-7494)$

Boehmite AlOOH (04-016-2858/01-073-9093)

Anorthite $\mathrm{CaAl}_{2} \mathrm{Si}_{2} \mathrm{O}_{8}(04-012-1276)$

Dmisteinbergite $\mathrm{CaAl}_{2} \mathrm{Si}_{2} \mathrm{O}_{8}$ (04-011-6236)

Portlandite $\mathrm{Ca}(\mathrm{OH})_{2}(00-050-0008)$

ron oxide hydroxide $\mathrm{FeOOH}$ (04-015-8205)

Calcium carbonate $\mathrm{CaCO}_{3}(01-081-2502)$
Perovskite $\mathrm{CaTiO}_{3}$ (00-022-0153)

Gehlenite $\mathrm{Al}_{2} \mathrm{Ca}_{2} \mathrm{O}_{7} \mathrm{Si}$ (04-013-2147)

Quartz $\mathrm{SiO}_{2}$ (01-085-0457)

Hematite $\mathrm{Fe}_{2} \mathrm{O}_{3}$ (04-003-1445)

Mullite $\mathrm{Al}\left(\mathrm{Al}_{0.69} \mathrm{Si}_{1.22} \mathrm{O}_{4.85}\right)(01-089-2644)$

Quartz $\mathrm{SiO}_{2}$ (04-018-0234)

Gehlenite $\mathrm{Al}_{2} \mathrm{Ca}_{2} \mathrm{O}_{7} \mathrm{Si}$ (04-015-7931)

Hematite $\mathrm{Fe}_{2} \mathrm{O}_{3}$ (01-089-0597)

Mullite $\mathrm{Al}\left(\mathrm{Al}_{0.69} \mathrm{Si}_{1.22} \mathrm{O}_{4 \cdot 85}\right)(01-089-2644)$

Gehlenite $\mathrm{Al}_{2} \mathrm{Ca}_{2} \mathrm{O}_{7} \mathrm{Si}$ (04-015-7930)

Quartz $\mathrm{SiO}_{2}$ (01-085-0798/04-007-1438/ 04-007-1808)

Perovskite $\mathrm{CaTiO}_{3}$ (01-073-9783)

Mullite $\mathrm{Al}\left(\mathrm{Al}_{0.69} \mathrm{Si}_{1.22} \mathrm{O}_{4.85}\right)(01-089-2644)$

Corundum $\mathrm{Al}_{2} \mathrm{O}_{3}$ (04-007-9625)

Quartz $\mathrm{SiO}_{2}$ (04-006-1757)

Brownmillerite $\mathrm{Ca}_{2} \mathrm{Fe}_{1 \cdot 63} \mathrm{Al}_{0.37} \mathrm{O}_{5}$ (04-015-8017)

Quartz $\mathrm{SiO}_{2}$ (04-015-7167)

Brownmillerite $\mathrm{Ca}_{2} \mathrm{Fe}_{1 \cdot 63} \mathrm{Al}_{0.37} \mathrm{O}_{5} / \mathrm{Ca}_{2} \mathrm{FeAlO}_{5}$ (04-015-8017/04-011-5939)

Quartz $\mathrm{SiO}_{2}$ (04-015-7167)

Brownmillerite $\mathrm{Ca}_{2} \mathrm{Fe}_{1.63} \mathrm{Al}_{0.37} \mathrm{O}_{5}$ (04-015-8017/04-011-5939)

Table 4. Continued 


\begin{tabular}{|c|c|c|c|c|c|c|}
\hline \multicolumn{6}{|c|}{$2 \theta:$ degrees } & \multirow{3}{*}{$\begin{array}{l}\text { Identified phase } \\
\text { (see Table } 4 \text { for ICDD numbers) }\end{array}$} \\
\hline \multicolumn{2}{|l|}{ Control } & \multicolumn{2}{|c|}{ Acid treated, core } & \multicolumn{2}{|c|}{ Acid treated, surface } & \\
\hline TSRC-1 & TSRC-2 & TSRC-1 & TSRC-2 & TSRC-1 & TSRC-2 & \\
\hline- & - & $12 \cdot 05$ & $12 \cdot 07$ & - & - & Dminsteinbergite \\
\hline- & - & $12 \cdot 47$ & - & $12 \cdot 47$ & - & Garronite \\
\hline $14 \cdot 58$ & $14 \cdot 44$ & $14 \cdot 56$ & $14 \cdot 46$ & $14 \cdot 55$ & $14 \cdot 59$ & Boehmite \\
\hline- & - & - & $14 \cdot 75$ & - & $14 \cdot 75$ & Bassanite \\
\hline- & - & - & - & $15 \cdot 41$ & $15 \cdot 54$ & Alunite \\
\hline- & - & - & $15 \cdot 86$ & - & - & Analcime \\
\hline- & - & $16 \cdot 48$ & $16 \cdot 3$ & $16 \cdot 35$ & $16 \cdot 35$ & Mullite \\
\hline $17 \cdot 62$ & $17 \cdot 46$ & - & $17 \cdot 56$ & - & - & Katoite \\
\hline- & - & $18 \cdot 02$ & - & $17 \cdot 88$ & $17 \cdot 98$ & Garronite/alunite \\
\hline- & - & - & $18 \cdot 34$ & - & - & Analcime \\
\hline- & - & $20 \cdot 10$ & $20 \cdot 08 / 20 \cdot 11$ & - & $20 \cdot 08$ & Dminsteinbergite/gypsum \\
\hline $20 \cdot 14$ & $20 \cdot 18$ & $20 \cdot 18$ & $20 \cdot 1$ & $20 \cdot 1$ & $20 \cdot 16$ & Quartz \\
\hline $20 \cdot 38$ & $20 \cdot 20$ & - & $20 \cdot 20$ & - & - & Katoite \\
\hline- & - & $21 \cdot 5$ & - & $21 \cdot 5$ & - & Garronite \\
\hline- & - & $22 \cdot 02$ & - & $22 \cdot 02$ & - & Garronite \\
\hline- & - & $23 \cdot 48$ & $23 \cdot 38$ & $23 \cdot 4$ & $23 \cdot 50$ & Dminsteinbergite \\
\hline- & - & $24 \cdot 18$ & $24 \cdot 12$ & $24 \cdot 0$ & $24 \cdot 1 / 24 \cdot 16$ & Dminsteinbergite/hematite \\
\hline- & - & - & $25 \cdot 50$ & - & $25 \cdot 42$ & Anhydrate \\
\hline $25 \cdot 74$ & - & $25 \cdot 74$ & - & $25 \cdot 56$ & $25 \cdot 48$ & Corundum/alunite \\
\hline- & - & - & $25 \cdot 86$ & - & $25 \cdot 76-26 \cdot 0$ & Bassanite \\
\hline- & - & $26 \cdot 08$ & $26 \cdot 05$ & - & - & Analcime \\
\hline $26 \cdot 32$ & $26 \cdot 22$ & $26 \cdot 32$ & $26 \cdot 2$ & $26 \cdot 19 / 26 \cdot 32$ & $25 \cdot 9-26 \cdot 3$ & Quartz/mullite \\
\hline- & - & $26 \cdot 62$ & - & $26 \cdot 62$ & $26 \cdot 6$ & Quartz \\
\hline $26 \cdot 98$ & $26 \cdot 86$ & $26 \cdot 98$ & $26 \cdot 99$ & - & - & Katoite \\
\hline- & - & $27 \cdot 8$ & - & - & - & Garronite \\
\hline $28 \cdot 16$ & $28 \cdot 18$ & $28 \cdot 12$ & $28 \cdot 1$ & $28 \cdot 18$ & $28 \cdot 18$ & Quartz \\
\hline $28 \cdot 29$ & $28 \cdot 23$ & $28 \cdot 29$ & $28 \cdot 14$ & $28 \cdot 29$ & $28 \cdot 22$ & Garronite/boehmite \\
\hline $28 \cdot 86$ & $28 \cdot 76$ & - & $28 \cdot 9 / 28 \cdot 84$ & - & - & Katoite/gypsum/quartz \\
\hline $29 \cdot 5$ & $29 \cdot 4$ & $29 \cdot 5$ & $29 \cdot 34$ & - & - & Calcium carbonate \\
\hline- & - & - & $29 \cdot 48$ & - & $29 \cdot 8-30 \cdot 2$ & Bassanite \\
\hline- & - & - & - & $29 \cdot 90$ & $30 \cdot 1$ & Alunite \\
\hline- & - & - & $30 \cdot 64 / 30 \cdot 3$ & - & $30 \cdot 36$ & Analcime/hematite \\
\hline- & - & - & $31 \cdot 34$ & - & $31 \cdot 36$ & Anhydrate \\
\hline- & - & - & - & $31 \cdot 36$ & $31 \cdot 37$ & Alunite \\
\hline- & $31 \cdot 40$ & - & $31 \cdot 40$ & - & $31 \cdot 4$ & Gehlenite \\
\hline- & - & $31 \cdot 63$ & $31 \cdot 58$ & - & $31 \cdot 62$ & Dminsteinbergite \\
\hline- & - & - & $31 \cdot 7$ & - & $31 \cdot 8$ & Bassanite \\
\hline $32 \cdot 38$ & $32 \cdot 24$ & - & $32 \cdot 39$ & - & - & Katoite \\
\hline $33 \cdot 28$ & $33 \cdot 12$ & $33 \cdot 26$ & $33 \cdot 14$ & $33 \cdot 08 / 33 \cdot 28$ & $33 \cdot 12 / 33 \cdot 3$ & Perovskite/iron oxide/mullite \\
\hline- & - & $33 \cdot 60$ & - & $33 \cdot 60$ & - & Garronite \\
\hline- & - & $35 \cdot 16$ & $35 \cdot 14$ & - & $35 \cdot 20$ & Dminsteinbergite \\
\hline $35 \cdot 22$ & $35 \cdot 4$ & $35 \cdot 22$ & $35 \cdot 2$ & $35 \cdot 22$ & $36 \cdot 2$ & Corundum/mullite \\
\hline $36 \cdot 1$ & $36 \cdot 1$ & $35 \cdot 78$ & $35 \cdot 9 / 35 \cdot 68$ & - & $35 \cdot 9-35 \cdot 8$ & Dminsteinbergite/hematite \\
\hline $37 \cdot 06$ & $36 \cdot 88$ & - & $37 \cdot 08$ & - & - & Katoite \\
\hline $37 \cdot 9$ & - & $37 \cdot 7$ & - & $37 \cdot 7$ & - & Corundum \\
\hline
\end{tabular}

Table 5. Identified XRD reflections in TSRC-1 and TSRC-2 blends (continued on next page) 
20: degrees

\begin{tabular}{|c|c|c|c|c|c|c|}
\hline \multicolumn{2}{|l|}{ Control } & \multicolumn{2}{|c|}{ Acid treated, core } & \multicolumn{2}{|c|}{ Acid treated, surface } & \multirow{2}{*}{ (see Table 4 for ICDD numbers) } \\
\hline TSRC-1 & TSRC-2 & TSRC-1 & TSRC-2 & TSRC-1 & TSRC-2 & \\
\hline $38 \cdot 42$ & $38 \cdot 3$ & 38.42 & $38 \cdot 33$ & 38.42 & $38 \cdot 36$ & Boehmite \\
\hline $39 \cdot 5$ & $39 \cdot 36$ & $39 \cdot 4$ & $39 \cdot 4$ & $39 \cdot 73$ & $39 \cdot 4-39 \cdot 8 / 39 \cdot 6$ & Alunite/calcium carbonate \\
\hline- & - & - & $39 \cdot 58$ & - & $39 \cdot 7$ & Bassanite \\
\hline- & - & $39 \cdot 82$ & $39 \cdot 88$ & $40 \cdot 0$ & $39 \cdot 72$ & Dminsteinbergite \\
\hline $39 \cdot 85$ & $39 \cdot 72$ & - & $39 \cdot 95$ & - & - & Katoite \\
\hline $41 \cdot 0$ & $41 \cdot 15$ & $40 \cdot 8 / 40 \cdot 87$ & $40 \cdot 8 / 40 \cdot 9$ & $40 \cdot 75$ & $40 \cdot 75 / 40 \cdot 8$ & $\begin{array}{l}\text { Iron oxide/calcium carbonate/ } \\
\text { mullite/anhydrate }\end{array}$ \\
\hline- & - & $42 \cdot 10$ & $42 \cdot 00$ & - & $42 \cdot 1$ & Dminsteinbergite \\
\hline- & - & $42 \cdot 71$ & $42 \cdot 63$ & - & $42 \cdot 75$ & Dminsteinbergite \\
\hline- & - & - & $42 \cdot 95$ & - & $42 \cdot 3-42 \cdot 8$ & Bassanite \\
\hline $43 \cdot 47$ & - & $43 \cdot 31$ & - & $43 \cdot 31$ & - & Corundum \\
\hline $45 \cdot 11$ & $45 \cdot 01$ & - & $45 \cdot 22$ & - & - & Katoite \\
\hline- & $47 \cdot 59$ & - & $47 \cdot 51 / 47 \cdot 24-47 \cdot 55$ & - & $47 \cdot 3-47 \cdot 4$ & Perovskite/calcium carbonate \\
\hline- & - & - & - & $47 \cdot 65$ & $47 \cdot 80$ & Alunite \\
\hline $48 \cdot 99$ & $48 \cdot 87$ & - & $48 \cdot 89$ & - & $48 \cdot 9-49 \cdot 2$ & Boehmite \\
\hline $49 \cdot 47$ & $49 \cdot 21$ & $49 \cdot 27-49 \cdot 40$ & $49 \cdot 33$ & $49 \cdot 27$ & $49 \cdot 2-49 \cdot 3$ & Dminsteinbergite/quartz/boehmite \\
\hline
\end{tabular}

Table 5. Continued

advanced argillic alterations after extreme base leaching and sulfate fixation by minerals upon encountering groundwater with a high sulfuric acid concentration (Kiyosaki et al., 2003; Steiner, 1968; Wohletz and Grant, 1992). Alunite has a low dissolution rate over a wide temperature range (Miller et al., 2016; Stoffregen et al., 1994).

For all other samples, calcium sulfates such as bassanite crystallised along with alunite in the surface layers. In the FAFrich mixes (TSRC-3 and TSRC-4), calcium-potassium sulfate, gorgeyite, was identified. Boehmite survived in the surfaces of all the TSRC samples along with non-reacted phases such as mullite, corundum, quartz, hematite, gehlenite and perovskite. Some anorthite, dmisteinbergite and zeolites remained as minor phases along with iron silicate, hedenbergite (TSRC-3).

The CPC sample partially lost calcium aluminium silicates and calcium phosphates from the cores of the exposed samples and their peaks almost disappeared in the acid-treated surfaces. An intermediate phase of apatite degradation, sulfonated hydroxylapatite (ICDD 04-013-7494), was identified in the core (split peaks at $2 \theta^{\circ}$ of $25 \cdot 9,29 \cdot 16,32 \cdot 0,40 \cdot 16$ ), while the major crystalline phases at the acid-attacked surfaces were non-reacted gehlenite and gypsum.

The class G/silica control sample showed peaks of non-reacted quartz, tobermorite 9A, portlandite or calcium carbonates and brownmillerite. After acid exposure, the crystalline composition of the sample surfaces mostly included gypsum along with non-reacted phases (quartz and brownmillerite). The cores of the samples had some tobermorite and calcium carbonate along with crystalline gypsum.

In summary, the XRD studies demonstrated the susceptibility of calcium-based crystalline phases to low-pH sulfuric acid attack. They transferred mostly into amorphous phases or crystalline calcium sulfates. These acid-sensitive phases included anorthite and dmisteinbergite, katoites, calcium phosphates, portlandite, tobermorite and calcium carbonates. All zeolites formed in FAF-containing blends were also susceptible to acid attack. The TSRC blends formed potassium aluminium sulfate, alunite, which persisted under the experimental conditions.

Analyses of degraded samples - $\mu$ EDX oxide composition Table 8 shows the oxide compositions of the blends averaged over 3 by $4 \mathrm{~mm}$ areas of ground samples. After the acid treatment, the silicon content of all the TSRC samples and the CPC increased at the surface. Calcium strongly decreased, especially for TSRC-1, which did not form any calcium sulfates, while the decline in aluminium content was less significant than that of calcium. Relating these data to the XRD results suggests that decomposition of calcium-(aluminium)silicate hydrates leads to calcium dissolution and partial precipitation of its sulfate salts when they reach their saturation level, polymerisation of remaining $-\mathrm{Si}-\mathrm{O}-$ under acidic 


\begin{tabular}{|c|c|c|c|c|c|c|}
\hline \multirow{2}{*}{\multicolumn{2}{|c|}{ Control }} & \multicolumn{4}{|c|}{$2 \theta:$ degrees } & \multirow{3}{*}{$\begin{array}{l}\text { Identified phase } \\
\text { (See Table } 4 \text { for ICDD numbers) }\end{array}$} \\
\hline & & \multicolumn{2}{|c|}{ Acid treated, core } & \multicolumn{2}{|c|}{ Acid treated, surface } & \\
\hline TSRC-3 & TSRC-4 & TSRC-3 & TSRC-4 & TSRC-3 & TSRC-4 & \\
\hline $12 \cdot 27$ & $12 \cdot 27$ & $12 \cdot 21$ & $12 \cdot 21$ & - & $12 \cdot 2$ & Gismondine \\
\hline $12 \cdot 47$ & $12 \cdot 48$ & $12 \cdot 43$ & $12 \cdot 47$ & - & $12 \cdot 4$ & $\begin{array}{l}\text { Garronite/harmotome/gobbinsite/ } \\
\text { phillipsite }\end{array}$ \\
\hline $14 \cdot 49$ & - & $14 \cdot 42$ & - & $14 \cdot 49$ & - & Boehmite \\
\hline- & - & $14 \cdot 73$ & $14 \cdot 84$ & $14 \cdot 73$ & $14 \cdot 71$ & Bassanite \\
\hline $15 \cdot 83$ & $15 \cdot 83$ & $15 \cdot 93$ & $15 \cdot 74$ & $15 \cdot 89$ & $15 \cdot 79$ & Analcime/gorgeyite \\
\hline $16 \cdot 43$ & $16 \cdot 48$ & $16 \cdot 32$ & $16 \cdot 38$ & $16 \cdot 42$ & $16 \cdot 38$ & Mullite/phillipsite \\
\hline $17 \cdot 84$ & $17 \cdot 7 / 17 \cdot 9$ & $17 \cdot 82$ & $17 \cdot 8$ & $17 \cdot 92$ & $17 \cdot 84$ & $\begin{array}{l}\text { Phillipsite/garronite/harmotome/ } \\
\text { gobbinsite/katoite/alunite }\end{array}$ \\
\hline $18 \cdot 04$ & $18 \cdot 04$ & $18 \cdot 06$ & 18.06 & - & - & Gismondine \\
\hline $18 \cdot 20$ & $18 \cdot 30$ & $18 \cdot 34$ & $18 \cdot 26$ & $18 \cdot 32$ & $18 \cdot 28$ & Analcime \\
\hline- & - & - & $20 \cdot 74$ & $20 \cdot 74$ & $20 \cdot 78$ & Bassanite \\
\hline $20 \cdot 88 / 20 \cdot 94$ & $20 \cdot 94$ & $20 \cdot 86 / 20 \cdot 92$ & $20 \cdot 84$ & $20 \cdot 86$ & $20 \cdot 86$ & Quartz/gismondine \\
\hline $21 \cdot 64$ & $21 \cdot 68$ & $21 \cdot 58$ & $21 \cdot 54$ & $21 \cdot 64$ & $21 \cdot 74$ & $\begin{array}{l}\text { Garronite/harmotome/gobbinsite/ } \\
\text { phillipsite }\end{array}$ \\
\hline $25 \cdot 6$ & - & $25 \cdot 6$ & - & $25 \cdot 46$ & $25 \cdot 38$ & Alunite/anhydrate/corundum \\
\hline- & - & $25 \cdot 54$ & $25 \cdot 68$ & $25 \cdot 68$ & $25 \cdot 68$ & Bassanite \\
\hline $25 \cdot 9 / 26 \cdot 2$ & $25 \cdot 99$ & $26 \cdot 16$ & $25 \cdot 94$ & $25 \cdot 9 / 26 \cdot 0$ & $25 \cdot 97$ & Mullite/analcime/calcium carbonate \\
\hline $26 \cdot 68$ & $26 \cdot 7$ & $26 \cdot 62$ & $26 \cdot 6$ & $26 \cdot 64$ & $26 \cdot 68$ & Quartz/gobbinsite/gismondine \\
\hline $27 \cdot 8$ & $27 \cdot 8$ & $27 \cdot 8$ & $27 \cdot 7$ & - & $27 \cdot 8$ & Garronite \\
\hline $\begin{array}{r}27 \cdot 86- \\
28 \cdot 12\end{array}$ & 28.00 & 28.04 & $28 \cdot 04$ & $27 \cdot 98$ & $28 \cdot 06$ & Anorthite/phillipsite/gismondine \\
\hline $28 \cdot 3$ & $28 \cdot 36$ & $28 \cdot 16$ & $28 \cdot 22$ & $28 \cdot 12 / 28 \cdot 22 / 28 \cdot 24$ & $28 \cdot 2$ & $\begin{array}{l}\text { Garronite/gobbinsite/harmotome/ } \\
\text { boehmite/gorgeyite }\end{array}$ \\
\hline $29 \cdot 42 / 29 \cdot 44$ & $29 \cdot 4$ & $29 \cdot 5$ & $29 \cdot 4-29 \cdot 8$ & $29 \cdot 42 / 29 \cdot 73$ & $29 \cdot 4-29 \cdot 8$ & $\begin{array}{l}\text { Gobbinsite/katoite/katoite silician } \\
\text { bassanite/calcium carbonate/ } \\
\text { gorgeyite/hedenbergite }\end{array}$ \\
\hline- & - & - & - & $29 \cdot 94$ & $29 \cdot 9$ & Alunite \\
\hline $30 \cdot 54$ & $30 \cdot 60$ & $30 \cdot 46$ & $30 \cdot 5$ & $30 \cdot 62$ & - & Analcime \\
\hline $30 \cdot 96$ & $31 \cdot 04$ & $30 \cdot 96$ & $30 \cdot 92$ & $30 \cdot 99$ & $30 \cdot 92$ & Mullite \\
\hline- & $31 \cdot 32$ & - & $31 \cdot 26$ & - & $31 \cdot 28$ & Gehlenite \\
\hline- & - & $31 \cdot 86$ & 31.94 & $31 \cdot 88 / 31 \cdot 92$ & $31 \cdot 82$ & Gorgeyite/bassanite \\
\hline $32 \cdot 86$ & $32 \cdot 70$ & $32 \cdot 6$ & $32 \cdot 68$ & - & - & $\begin{array}{l}\text { Gobbinsite/phillipsite/gismondine/ } \\
\text { perovskite/katoite/katoite silician/ } \\
\text { calcium carbonate }\end{array}$ \\
\hline $33 \cdot 24 / 33 \cdot 3$ & $33 \cdot 3$ & $33 \cdot 2 / 33 \cdot 3$ & $33 \cdot 2 / 33 \cdot 3$ & $33 \cdot 2$ & $33 \cdot 1$ & Mullite/hematite/phillipsite \\
\hline $33 \cdot 4-33 \cdot 52$ & $33 \cdot 4$ & $33 \cdot 5$ & $33 \cdot 4$ & - & $33 \cdot 5$ & Garronite/harmotome/gobbinsite \\
\hline $35 \cdot 20$ & - & $35 \cdot 04$ & - & $35 \cdot 14$ & - & Hedenbergite/corundum \\
\hline $35 \cdot 27$ & $35 \cdot 22$ & $35 \cdot 3$ & $35 \cdot 23$ & $35 \cdot 26$ & $35 \cdot 18$ & Mullite \\
\hline $35 \cdot 58$ & - & $35 \cdot 5$ & - & - & - & Gobbinsite \\
\hline $35 \cdot 74$ & $35 \cdot 87$ & $35 \cdot 67$ & $35 \cdot 68$ & $35 \cdot 76$ & $35 \cdot 68$ & Hematite/analcime \\
\hline $36 \cdot 6-37 \cdot 1$ & $36 \cdot 9$ & $37 \cdot 01$ & $36 \cdot 94$ & $37 \cdot 0$ & $36 \cdot 91$ & Gobbinsite/mullite \\
\hline $37 \cdot 8$ & - & $37 \cdot 84$ & - & $37 \cdot 76$ & - & $\begin{array}{l}\text { Corundum/gobbinsite/calcium } \\
\text { carbonate }\end{array}$ \\
\hline
\end{tabular}

Table 6. Identified XRD reflections in TSRC-3 and TSRC-4 blends (continued on next page) 
$2 \theta$ : degrees

\begin{tabular}{|c|c|c|c|c|c|c|}
\hline \multicolumn{2}{|l|}{ Control } & \multicolumn{2}{|c|}{ Acid treated, core } & \multicolumn{2}{|c|}{ Acid treated, surface } & \\
\hline TSRC-3 & TSRC-4 & TSRC-3 & TSRC-4 & TSRC-3 & TSRC-4 & \\
\hline $38 \cdot 3$ & 38.43 & $38 \cdot 40$ & 38.43 & $38 \cdot 3$ & $38 \cdot 3$ & $\begin{array}{l}\text { Boehmite/gobbinsite/calcium } \\
\text { carbonate }\end{array}$ \\
\hline $39 \cdot 34$ & $39 \cdot 22$ & $39 \cdot 36$ & $39 \cdot 3$ & $39 \cdot 24$ & $39 \cdot 11$ & Mullite \\
\hline $39 \cdot 46$ & - & $39 \cdot 5$ & $39 \cdot 4$ & $39 \cdot 4$ & $39 \cdot 4$ & Quartz \\
\hline- & - & $39 \cdot 64$ & $39 \cdot 6$ & 39.66 & $39 \cdot 6$ & Bassanite \\
\hline- & - & - & - & $39 \cdot 76$ & $39 \cdot 70$ & Alunite \\
\hline $40 \cdot 57$ & $40 \cdot 55$ & $40 \cdot 58$ & $40 \cdot 41$ & - & - & Katoite/katoite silician/analcime \\
\hline $40 \cdot 85$ & $40 \cdot 89$ & $40 \cdot 83$ & $40 \cdot 81$ & $40 \cdot 77$ & $40 \cdot 77$ & Mullite/gobbinsite \\
\hline $40 \cdot 98$ & $40 \cdot 8$ & $40 \cdot 8$ & $40 \cdot 8$ & $40 \cdot 81 / 40 \cdot 97$ & $40 \cdot 8$ & Bassanite/hematite \\
\hline $42 \cdot 51$ & $42 \cdot 61$ & $42 \cdot 21 / 42 \cdot 45$ & $42 \cdot 0-42 \cdot 7$ & $42 \cdot 23 / 42 \cdot 49 / 42 \cdot 67$ & $42 \cdot 0-42 \cdot 7$ & $\begin{array}{l}\text { Hedenbergite/diopside/bassanite/ } \\
\text { mullite/gobbinsite }\end{array}$ \\
\hline $43 \cdot 35$ & - & $43 \cdot 37$ & - & $43 \cdot 33$ & - & Corundum \\
\hline $45 \cdot 91-46 \cdot 3$ & $45 \cdot 97$ & $45 \cdot 89-45 \cdot 91$ & $45 \cdot 81$ & $45 \cdot 75$ & $45 \cdot 77$ & $\begin{array}{l}\text { Katoite/katoite silician/gobbinsite/ } \\
\text { garronite/calcium carbonate }\end{array}$ \\
\hline- & $47 \cdot 72$ & $47 \cdot 59$ & $47 \cdot 6$ & $47 \cdot 65 / 47 \cdot 91$ & $47 \cdot 75$ & Bassanite/alunite/gorgeyite/perovskite \\
\hline $48 \cdot 9-49 \cdot 5$ & - & 48.93 & - & $48 \cdot 79$ & - & Anhydrate/boehmite/gobbinsite \\
\hline $49 \cdot 3$ & - & $49 \cdot 29$ & $49 \cdot 27$ & $48 \cdot 81-49 \cdot 49 / 49 \cdot 51$ & $49 \cdot 23 / 49 \cdot 4$ & Bassanite/boehmite/gorgeyite \\
\hline $50 \cdot 11$ & $50 \cdot 11 / 50 \cdot 27$ & $50 \cdot 07$ & $50 \cdot 1$ & $50 \cdot 09-50 \cdot 27$ & $50 \cdot 07$ & Gismondine/quartz/bassanite \\
\hline
\end{tabular}

Table 6. Continued

conditions into an amorphous gel that stays on the surface, and the formation of amorphous or crystalline (alunite) phases involving aluminium. Correspondently, the formulations with higher calcium contents (class G/silica, TSRC-2, TSRC-4 and CPC) have increased precipitation of calcium sulfates, forming a surface layer that controls sulfur concentrations and limits its further permeation $(4 \cdot 3 \%, 2 \cdot 7 \%, 2 \cdot 3 \%$ and $0 \%$ core sulfur in the class G/silica, TSRC-2, TSRC-4 and CPC blends respectively). With the exception of the class G/silica blend, this layer was not sufficient to prevent weight and diameter losses of the samples (Table 3).

In aluminium-rich TSRC-1, the surface sulfur-poor solution concentration is probably controlled by alunite and sulfurreacting aluminium-rich amorphous phases, resulting in its absence in the sample's core. Relatively high sulfur permeation into the core of FAF- and aluminium-rich TSRC-3 could be a result of low calcium content, and the presence of non-reacted FAF that would increase permeability and acid sensitivity of both FAF-rich samples (TSRC-3 and TSRC-4). In these formulations, the sulfur surface content is lower than in the CAC-rich counterparts (TSRC-1 and TSRC-2), suggesting partial removal of sulfates. This is in agreement with the smaller corrosion depth, higher weight loss and more significant diameter decrease in comparison with TSRC-1 and 2 (Table 3).
The EDX measurements confirm the elimination of calcium phosphates from the CPC under acid attack (73\% and 83\% of phosphorous is lost from the surface and the core, respectively).

\section{Analyses of degraded samples - differential thermal gravimetric (DTG) analyses}

Figure 5 shows DTG analyses of the cement blends. For the TSRC samples, the weight losses below about $250^{\circ} \mathrm{C}$ are associated with calcium-sodium-aluminium-silicate hydrates (mostly probably amorphous from fly ash F and CAC) (Foldvari, 2011; Winnefeld et al., 2010) and zeolite-related weight losses (peaks at $\sim 60-90^{\circ} \mathrm{C}, \sim 150-230^{\circ} \mathrm{C}$ ). The weight loss at $\sim 280^{\circ} \mathrm{C}$ is due to the decomposition of katoite with a possible contribution of zeolites $\left(\sim 270-360^{\circ} \mathrm{C}\right)$ and that at $\sim 500^{\circ} \mathrm{C}$ from boehmite with a possible contribution of analcime (Harada et al., 1972). The losses above $\sim 600^{\circ} \mathrm{C}$ are from carbonates from the blend components or formed during mixing of the slurries and the weight losses at temperatures above about $800^{\circ} \mathrm{C}$ were attributed to non-reacted fly ash (Pyatina and Sugama, 2016). The flu ash volume fraction increased after the erosion of calcium-containing phases, as did the high-temperature weight loss peak on the thermograms of acid-treated samples.

The control sample of TSRC-1 had a narrow DTG peak at $\sim 81^{\circ} \mathrm{C}$, likely due to the decomposition of hydroxysodalite. In 


\begin{tabular}{|c|c|c|c|c|c|c|}
\hline \multirow{2}{*}{\multicolumn{2}{|c|}{ Control }} & \multicolumn{2}{|c|}{$2 \theta:$ degrees } & & & \multirow{3}{*}{$\begin{array}{l}\text { Identified phase } \\
\text { (see Table } 4 \text { for ICDD numbers) }\end{array}$} \\
\hline & & \multicolumn{2}{|c|}{ Acid treated, core } & \multicolumn{2}{|c|}{ Acid treated, surface } & \\
\hline CPC & G/silica & CPC & G/silica & CPC & G/silica & \\
\hline- & - & - & - & $11 \cdot 66$ & $11 \cdot 61$ & Gypsum \\
\hline 11.96 & - & 11.98 & - & - & - & Dmisteinbergite \\
\hline- & $12 \cdot 19$ & - & $12 \cdot 34$ & - & - & Brownmillerite \\
\hline $14 \cdot 52$ & - & $14 \cdot 64$ & - & $14 \cdot 52$ & - & Boehmite \\
\hline $16 \cdot 22$ & - & - & - & $16 \cdot 3$ & - & Mullite \\
\hline $17 \cdot 54$ & - & - & - & - & - & Katoite silician \\
\hline- & - & - & $17 \cdot 78$ & - & - & Iron oxide hydroxide \\
\hline- & 18.04 & - & - & - & - & Tobermorite 9A \\
\hline $20 \cdot 00$ & - & $20 \cdot 24$ & - & $20 \cdot 06$ & - & Dmisteinbergite \\
\hline- & $20 \cdot 88$ & - & $21 \cdot 06 / 21 \cdot 12$ & $20 \cdot 78$ & $20 \cdot 68$ & $\begin{array}{l}\text { Gypsum/quartz/iron oxide } \\
\text { hydroxide }\end{array}$ \\
\hline- & $23 \cdot 12$ & - & $23 \cdot 12$ & - & - & Brownmillerite \\
\hline $23 \cdot 38$ & - & $23 \cdot 62$ & - & $23 \cdot 37$ & $23 \cdot 38$ & Dmisteinbergite/quartz/gypsum \\
\hline $24 \cdot 1$ & - & $24 \cdot 32$ & - & $24 \cdot 18$ & - & Dmisteinbergite/hematite \\
\hline- & $24 \cdot 94 / 24.96$ & - & $25 \cdot 16$ & - & - & $\begin{array}{l}\text { Tobermorite 9A/calcium } \\
\text { carbonate }\end{array}$ \\
\hline $25 \cdot 92 / 26 \cdot 6$ & - & $26 \cdot 14$ & - & $26 \cdot 02$ & - & $\begin{array}{l}\text { Mullite/hydroxylapatite/analcime/ } \\
\text { carbonated hydroxylapatite/ } \\
\text { hydroxylapatite sulfonated }\end{array}$ \\
\hline- & $26 \cdot 34$ & - & - & - & & Portlandite \\
\hline $26 \cdot 6 / 26 \cdot 82$ & $26 \cdot 66$ & - & $26 \cdot 84$ & $26 \cdot 64$ & $26 \cdot 64$ & $\begin{array}{l}\text { Anorthite/calcium hydrogen } \\
\text { phosphate/quartz }\end{array}$ \\
\hline- & $26 \cdot 92-27 \cdot 22$ & - & $27 \cdot 16-27 \cdot 6$ & - & - & Calcium carbonate \\
\hline $27 \cdot 88 / 28 \cdot 1$ & - & $28 \cdot 34$ & - & $28 \cdot 16 / 28 \cdot 14$ & - & Anorthite/boehmite \\
\hline 28.93 & - & $28 \cdot 9$ & - & - & - & $\begin{array}{l}\text { Hydroxylapatite/katoite silician/ } \\
\text { portlandite }\end{array}$ \\
\hline $29 \cdot 2$ & $29 \cdot 16$ & $29 \cdot 16$ & $29 \cdot 24$ & $29 \cdot 11 / 29 \cdot 12$ & $28 \cdot 12 / 29 \cdot 11$ & $\begin{array}{l}\text { Hydroxylapatite sulfonated/ } \\
\text { gypsum/calcium carbonate }\end{array}$ \\
\hline $29 \cdot 22$ & $29 \cdot 44$ & $29 \cdot 6$ & $29 \cdot 56$ & - & - & $\begin{array}{l}\text { Boehmite/tobermorite 9A/calcium } \\
\text { carbonate/quartz }\end{array}$ \\
\hline $30 \cdot 18$ & - & - & - & - & - & $\begin{array}{l}\text { Anorthite/calcium hydrogen } \\
\text { phosphate }\end{array}$ \\
\hline- & - & - & - & $31 \cdot 12$ & $31 \cdot 18$ & Gypsum \\
\hline $31 \cdot 28$ & - & $31 \cdot 36$ & - & - & - & Gehlenite \\
\hline $31 \cdot 5$ & - & $31 \cdot 47$ & - & $31 \cdot 59$ & & Dmisteinbergite \\
\hline $31 \cdot 6$ & - & $31 \cdot 76$ & - & - & - & Hydroxylapatite/dmisteinbergite \\
\hline 31.94 & $31 \cdot 9$ & - & - & - & - & $\begin{array}{l}\text { Carbonated hydroxylapatite/ } \\
\text { portlandite }\end{array}$ \\
\hline $32 \cdot 18$ & - & $32 \cdot 02$ & - & - & $32 \cdot 1$ & $\begin{array}{l}\text { Hydroxylapatite/carbonated } \\
\text { hydroxylapatite/hydroxylapatite } \\
\text { sulfonated/gypsum }\end{array}$ \\
\hline- & $32 \cdot 2 / 32 \cdot 36$ & - & $32 \cdot 44$ & - & $32 \cdot 15$ & Tobermorite 9A/brownmillerite \\
\hline $32 \cdot 48 / 32 \cdot 8$ & $32 \cdot 64$ & $32 \cdot 46$ & - & - & - & $\begin{array}{l}\text { Calcium hydrogen phosphate/ } \\
\text { katoite silician/portlandite }\end{array}$ \\
\hline
\end{tabular}

Table 7. Identified XRD reflections in CPC and class G/silica blends (continued on next page) 
$2 \theta$ : degrees

\begin{tabular}{|c|c|c|c|c|c|c|}
\hline \multicolumn{2}{|l|}{ Control } & \multicolumn{2}{|c|}{ Acid treated, core } & \multicolumn{2}{|c|}{ Acid treated, surface } & \multirow{2}{*}{ ee lable 4 for ICDD numbers) } \\
\hline CPC & G/silica & CPC & G/silica & CPC & G/silica & \\
\hline $33 \cdot 16$ & $32 \cdot 68-33 \cdot 0$ & $33 \cdot 21$ & $33-33 \cdot 6 / 33 \cdot 18$ & $33 \cdot 23$ & - & $\begin{array}{l}\text { Carbonated hydroxylapatite/ } \\
\text { perovskite/hematite/calcium } \\
\text { carbonate/iron oxide hydroxide }\end{array}$ \\
\hline- & - & 33.42 & $33 \cdot 4$ & 33.41 & $33 \cdot 34$ & Gypsum \\
\hline - & 33.58 & - & 33.70 & - & - & Tobermorite $9 \mathrm{~A}$ \\
\hline - & $33 \cdot 88 / 34 \cdot 1$ & - & $33 \cdot 7 / 34 \cdot 1$ & - & 33.52 & Brownmillerite \\
\hline $35 \cdot 06$ & - & $35 \cdot 28$ & - & $35 \cdot 20$ & - & Mullite/dmisteinbergite \\
\hline $35 \cdot 6 / 35 \cdot 7$ & - & $35 \cdot 86$ & - & $35 \cdot 74$ & - & $\begin{array}{l}\text { Katoite silician/calcium carbonate/ } \\
\text { hematite }\end{array}$ \\
\hline- & - & - & - & - & $35 \cdot 96 / 36 \cdot 7 / 37 \cdot 3$ & Gypsum \\
\hline $35 \cdot 9 / 38 \cdot 3$ & $36 \cdot 06$ & $37 \cdot 34 / 38 \cdot 43$ & $36 \cdot 8$ & $38 \cdot 36$ & - & $\begin{array}{l}\text { Boehmite/quartz/calcium } \\
\text { carbonate/iron oxide hydroxide }\end{array}$ \\
\hline $39 \cdot 23$ & $39 \cdot 23$ & - & - & - & - & Portlandite \\
\hline $39 \cdot 6$ & - & $39 \cdot 76$ & - & $39 \cdot 8$ & - & Dmisteinbergite \\
\hline $39 \cdot 68$ & - & $39 \cdot 98 / 40 \cdot 16$ & - & - & - & $\begin{array}{l}\text { Hydroxylapatite/hydroxylapatite } \\
\text { sulfonated }\end{array}$ \\
\hline $40 \cdot 83$ & - & 40.93 & - & $40 \cdot 74$ & $40 \cdot 73$ & Mullite/gypsum \\
\hline $41 \cdot 95$ & - & $42 \cdot 21$ & - & $42 \cdot 3$ & - & $\begin{array}{l}\text { Dmisteinbergite/calcium } \\
\text { carbonate }\end{array}$ \\
\hline $42 \cdot 59$ & & $42 \cdot 81$ & - & $42 \cdot 53$ & - & Mullite/dmisteinbergite \\
\hline- & - & - & - & $43 \cdot 3-43 \cdot 6$ & $43 \cdot 39-43 \cdot 57$ & Gypsum \\
\hline- & $43 \cdot 25 / 43 \cdot 9$ & - & $44 \cdot 3$ & - & $44 \cdot 23$ & Calcium carbonate/gypsum \\
\hline- & 44.05 & - & $44 \cdot 0$ & - & - & Tobermorite 9A \\
\hline $45 \cdot 15$ & - & $45 \cdot 15$ & - & - & - & Katoite silician \\
\hline- & - & - & $45 \cdot 7$ & - & 45.47 & Gypsum \\
\hline- & 46.99 & - & 46.99 & - & - & Portlandite \\
\hline- & $47 \cdot 45$ & - & $47 \cdot 4$ & - & $47 \cdot 5$ & $\begin{array}{l}\text { Brownmillerite/iron oxide } \\
\text { hydroxide }\end{array}$ \\
\hline $47 \cdot 55$ & $47 \cdot 7$ & $47 \cdot 77$ & - & $47 \cdot 79$ & - & $\begin{array}{l}\text { Calcium hydrogen phosphate/ } \\
\text { perovskite/calcium carbonate }\end{array}$ \\
\hline $47 \cdot 83$ & - & - & - & 47.79 & 47.83 & Dmisteinbergite/gypsum \\
\hline $47 \cdot 87$ & - & $48 \cdot 07 / 48 \cdot 21$ & - & $48 \cdot 37$ & $48 \cdot 37$ & Hydroxylapatite/gypsum \\
\hline $48 \cdot 49 / 48 \cdot 91$ & $48 \cdot 7$ & $48 \cdot 9$ & - & 48.9 & - & $\begin{array}{l}\text { Boehmite/quartz/calcium } \\
\text { carbonate }\end{array}$ \\
\hline $49 \cdot 03$ & - & 49.05 & - & - & - & Calcium hydrogen phosphate \\
\hline $49 \cdot 27$ & - & - & - & - & - & $\begin{array}{l}\text { Hydroxylapatite/carbonated } \\
\text { Hydroxylapatite }\end{array}$ \\
\hline $49 \cdot 39 / 49 \cdot 51$ & - & 49.53 & - & $49 \cdot 4$ & - & $\begin{array}{l}\text { Dmisteinbergite/hydroxylapatite/ } \\
\text { calcium carbonate/hematite }\end{array}$ \\
\hline- & $49 \cdot 95 / 50 \cdot 15$ & - & $49 \cdot 93 / 50 \cdot 35$ & $50 \cdot 27$ & $50 \cdot 23$ & $\begin{array}{l}\text { Tobermorite 9A/Gypsum/quartz/ } \\
\text { calcium carbonate/ } \\
\text { brownmillerite }\end{array}$ \\
\hline
\end{tabular}

Table 7. Continued

agreement with the XRD data, this peak was absent from the TSRC-2 thermogram. Both TSRC-1 and TSRC-2 showed DTG peaks at $\sim 236^{\circ} \mathrm{C}$ from various zeolites. The katoite peak was more significant for TSRC-2 than for TSRC-1, while boehmite peaks at $\sim 500^{\circ} \mathrm{C}$ were similar for both formulations, corresponding to $2 \cdot 2-2 \cdot 3 \%$ of weight loss. The peaks between 


\begin{tabular}{|c|c|c|c|c|c|c|}
\hline & Control & Core & Surface & Control & Core & Surface \\
\hline & \multicolumn{3}{|c|}{ TSRC-1 } & \multicolumn{3}{|c|}{ TSRC-2 } \\
\hline Aluminium oxide & 48 & 49 & 48 & 35 & 32 & 21 \\
\hline Silicon dioxide & 25 & 24 & 28 & 23 & 24 & 36 \\
\hline Calcium oxide & 19 & 21 & $1 \cdot 5$ & 32 & 33 & 11 \\
\hline Iron oxide & $4 \cdot 3$ & $4 \cdot 8$ & $4 \cdot 9$ & $6 \cdot 1$ & $6 \cdot 0$ & $7 \cdot 6$ \\
\hline Potassium oxide & $2 \cdot 0$ & $1 \cdot 0$ & $3 \cdot 3$ & $1 \cdot 3$ & - & $2 \cdot 7$ \\
\hline Titanium dioxide & $0 \cdot 80$ & - & $1 \cdot 0$ & $2 \cdot 3$ & $2 \cdot 7$ & $3 \cdot 2$ \\
\hline \multirow[t]{2}{*}{ Sulfur trioxide } & - & - & 14 & - & $2 \cdot 7$ & 18 \\
\hline & \multicolumn{3}{|c|}{ TSRC-3 } & \multicolumn{3}{|c|}{ TSRC-4 } \\
\hline Aluminium oxide & 41 & 41 & 38 & 29 & 30 & 18 \\
\hline Silicon dioxide & 35 & 31 & 35 & 35 & 32 & 48 \\
\hline Calcium oxide & 13 & 12 & $6 \cdot 4$ & 24 & 25 & 13 \\
\hline Iron oxide & $6 \cdot 6$ & $6 \cdot 2$ & $5 \cdot 5$ & $7 \cdot 4$ & $8 \cdot 1$ & $6 \cdot 5$ \\
\hline Potassium oxide & $2 \cdot 7$ & $2 \cdot 3$ & $2 \cdot 6$ & $3 \cdot 0$ & $2 \cdot 5$ & 1.9 \\
\hline Titanium dioxide & $1 \cdot 3$ & $1 \cdot 1$ & $1 \cdot 0$ & $2 \cdot 1$ & $2 \cdot 5$ & $2 \cdot 8$ \\
\hline \multirow[t]{2}{*}{ Sulfur trioxide } & - & $7 \cdot 0$ & 11 & - & $2 \cdot 3$ & 13 \\
\hline & \multicolumn{3}{|c|}{ CPC } & \multicolumn{3}{|c|}{ Class G/silica } \\
\hline Aluminium oxide & 29 & 35 & 27 & $2 \cdot 9$ & - & - \\
\hline Silicon dioxide & 18 & 21 & 31 & 29 & 30 & 15 \\
\hline Calcium oxide & 29 & 32 & 17 & 59 & 60 & 38 \\
\hline Phosphorus pentoxide & 15 & $2 \cdot 6$ & $4 \cdot 0$ & - & - & - \\
\hline Iron oxide & $5 \cdot 1$ & $5 \cdot 5$ & $6 \cdot 3$ & $5 \cdot 5$ & $5 \cdot 7$ & $1 \cdot 2$ \\
\hline Potassium oxide & 1.9 & 0.8 & - & 0.9 & - & - \\
\hline Titanium dioxide & $2 \cdot 0$ & $2 \cdot 2$ & $2 \cdot 7$ & $0 \cdot 2$ & $0 \cdot 3$ & - \\
\hline Sulfur trioxide & - & - & 12 & $2 \cdot 8$ & $4 \cdot 3$ & 46 \\
\hline \multicolumn{7}{|c|}{$\begin{array}{l}\text { Table 8. Oxide composition (in weight \%) of samples after } 18 \mathrm{~d} \\
\text { exposure to sulfuric acid at } 90^{\circ} \mathrm{C}(\mathrm{pH} 2 \cdot 5 \text { for } 6 \mathrm{~d} \text { followed by } \\
\mathrm{pH} 0.5 \text { for } 12 \mathrm{~d} \text { ) }\end{array}$} \\
\hline
\end{tabular}

$675^{\circ} \mathrm{C}$ and $680^{\circ} \mathrm{C}$ were from carbonates and represented less than $2 \%$ of the total weight loss.

The most noticeable new peaks after acid exposure were in the $710-730^{\circ} \mathrm{C}$ range from potassium aluminium sulfate, alunite (Foldvari, 2011), representing $4 \cdot 1 \%$ and $5 \cdot 8 \%$ weight losses for TSRC-1 and TSRC-2, respectively. In addition, there was a small peak at $\sim 412^{\circ} \mathrm{C}$ on the thermogram of TSRC-2, possibly related to calcium hydroxide (Nuruddeen, 2014). In agreement with the XRD results, the DTG peaks of zeolites and katoite clearly decreased for both TSRC-1 and TSRC-2 ( $\sim 30 \%$ reduction) while sulfate weight losses appeared (small weight loss in TSRC-1 samples at $\sim 115^{\circ} \mathrm{C}$ and at $120^{\circ} \mathrm{C}$ for TSRC-2).
The increase of FAF in TSRC-3 and TSRC-4 increased losses below $250^{\circ} \mathrm{C}$, associated with zeolites and calcium(sodium)-aluminium-silicate (C,N-A-S) hydrates $(8.4 \%$ and $8 \%$ for TSRC-3 and TSRC-4 compared with $\sim 5 \cdot 7 \%$ and $5 \cdot 2 \%$ for TSRC- 1 and TSRC-2), and decreased the katoite weight loss at $\sim 280^{\circ} \mathrm{C}$, which became only a shoulder on the peaks of zeolites decompositions. Boehmite decomposition at $\left(\sim 500^{\circ} \mathrm{C}\right.$ and $508^{\circ} \mathrm{C}$ for TSRC-3 and TSRC-4, respectively) was more clearly resolved for TSRC-3 containing more aluminium than for TSRC-4, where no clear peak of boehmite decomposition could be registered.

Boehmite losses increased by $\sim 20 \%$ at the acid-attacked surfaces of TSRC- 3 and the new peaks at $\sim 410(404)^{\circ} \mathrm{C}$ and 684 $(689)^{\circ} \mathrm{C}$ for $\mathrm{TSRC}-3$ (TSRC-4) could be attributed to calcium 

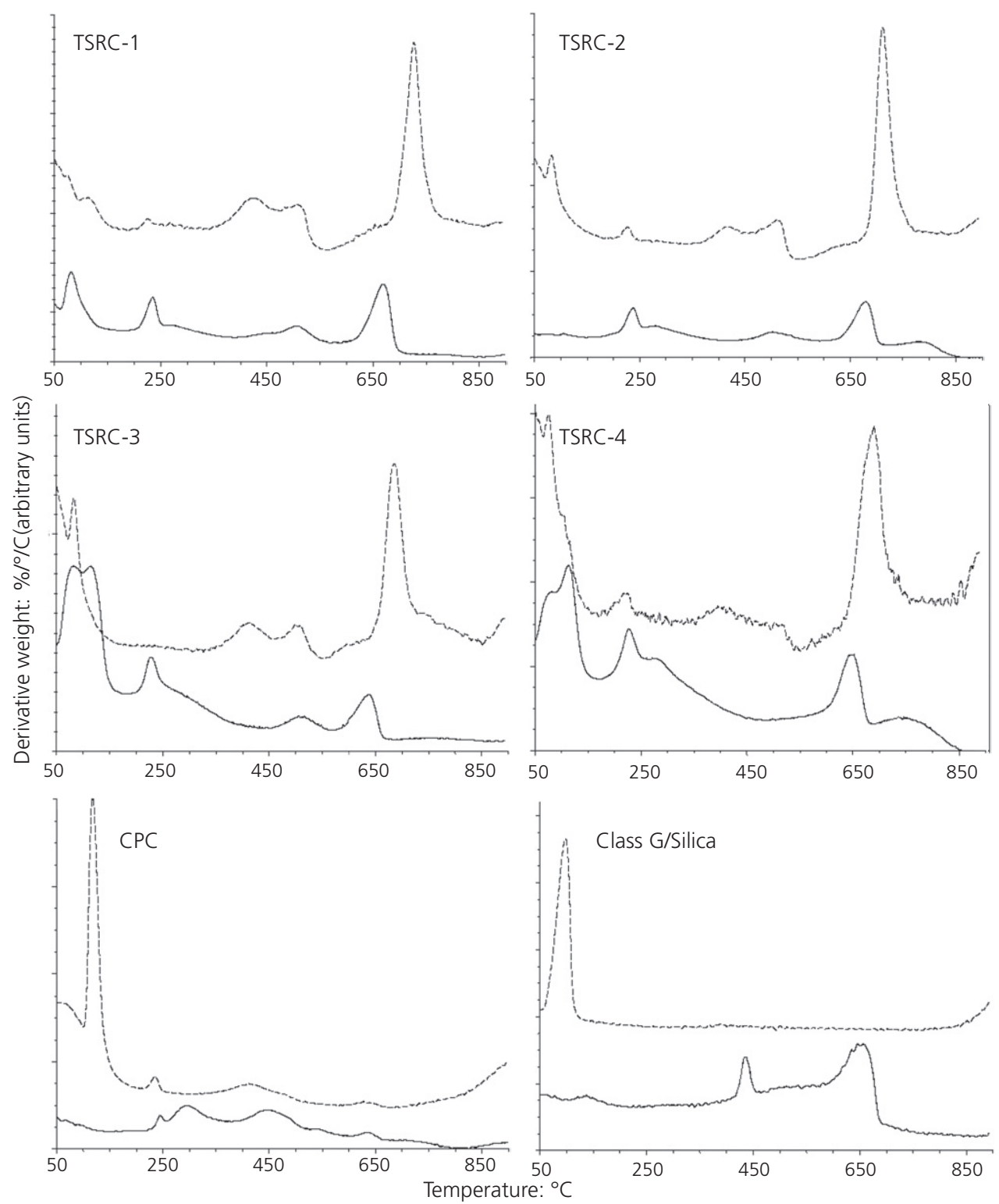

Figure 5. DTG analyses of cement blends. The lower curves are the control samples and the upper curves are for surfaces of the samples exposed to sulfuric acid

hydroxide and alunite, respectively. The broad band between $\sim 350^{\circ} \mathrm{C}$ and $500^{\circ} \mathrm{C}$ on the thermogram of TSRC-4 was possibly associated with overlapping calcium hydroxide and analcime decompositions. Confirming the XRD data, katoite disappeared from the surface and the weight losses associated with zeolites and amorphous C,N-A-S hydrates decreased conspicuously after the acid exposure (by $\sim 36 \%$ and $27 \%$ for TSRC-3 and TSRC-4, respectively). The decomposition of sulfates indicated by the XRD patterns, possibly due to their low crystallinity, happened at lower than expected temperatures, giving shoulders on the peak at $\sim 81^{\circ} \mathrm{C}$ (TSRC-3) and $\sim 103^{\circ} \mathrm{C}$ (TSRC-4).
The control CPC sample showed weight losses of amorphous hydrates and zeolites (DTG peaks at $\sim 66^{\circ} \mathrm{C}$ and $90^{\circ} \mathrm{C}$ ), further losses associated with zeolites at $\sim 185^{\circ} \mathrm{C}$ and $245^{\circ} \mathrm{C}$, followed by weight loss of katoite and analcime at $\sim 294^{\circ} \mathrm{C}$ $(\sim 2 \%)$. This was followed by weight loss of portlandite decomposition with a possible analcime contribution at $\sim 449^{\circ} \mathrm{C}$ (Harada et al., 1972), boehmite decomposition at $541^{\circ} \mathrm{C}$ and carbonate and non-reacted FAF decompositions at $>600^{\circ} \mathrm{C}$. After the acid exposure, the gypsum decomposition at $\sim 116^{\circ} \mathrm{C}(\sim 5 \%)$ completely dominated the small weight losses of residual zeolites $\left(\sim 235^{\circ} \mathrm{C}\right)$ and calcium hydroxide $\left(\sim 412^{\circ} \mathrm{C}\right)$. 

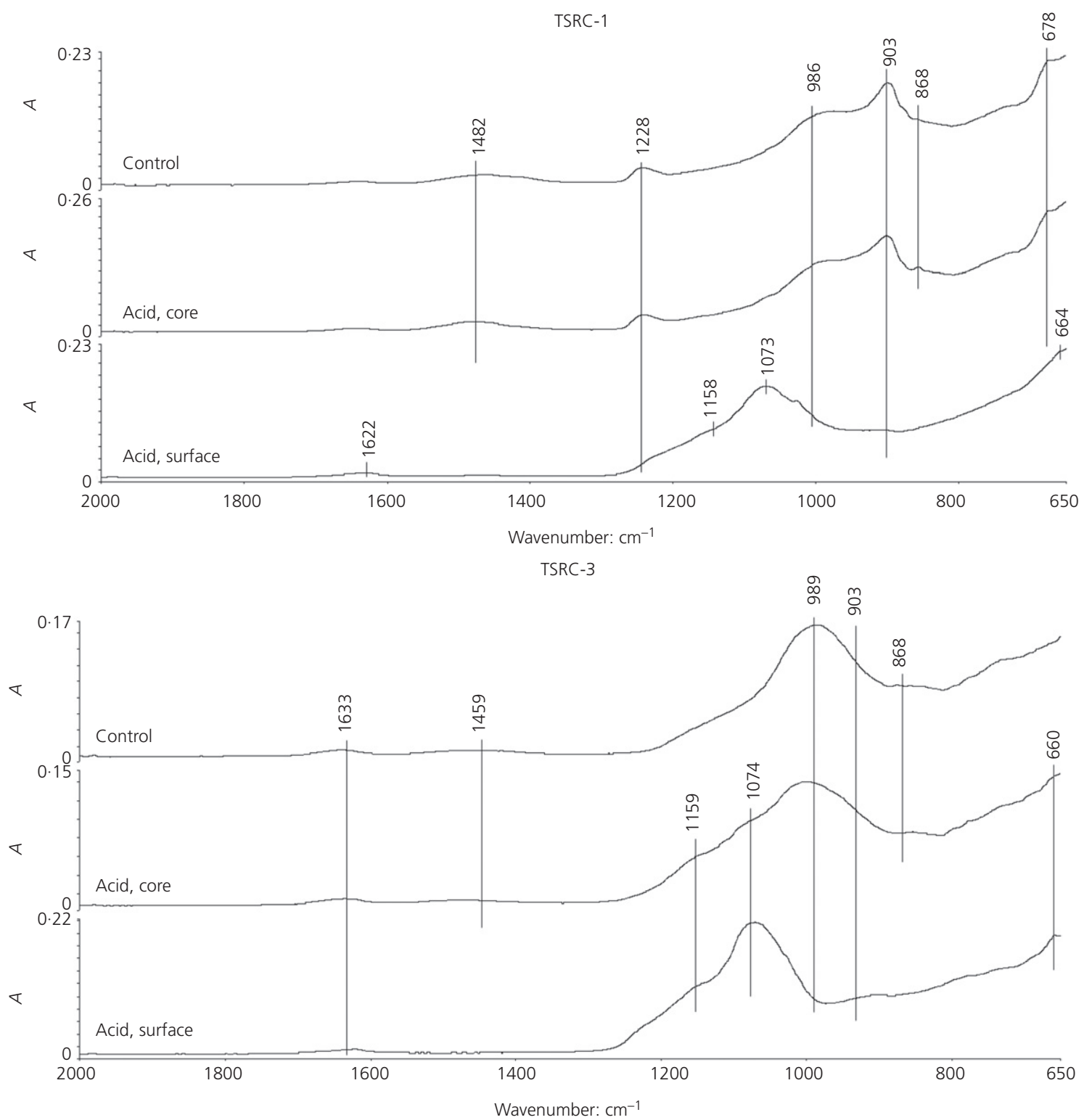

Figure 6. FTIR spectra of TSRC-1 and TSRC-3 blends before and after sulfuric acid exposure

The class G/silica blend weight losses after curing occurred below $\sim 100^{\circ} \mathrm{C}$ for calcium-silicate hydrates, at $\sim 136^{\circ} \mathrm{C}$ for gypsum, in a broad band between $\sim 250^{\circ} \mathrm{C}$ and $350^{\circ} \mathrm{C}$ for tobermorite and in a much shorter temperature range $\left(\sim 435^{\circ} \mathrm{C}\right)$ for portlandite; the weight loss above $\sim 850^{\circ} \mathrm{C}$ was not identified. In agreement with XRD and EDX measurements, the acid-exposed surface showed just one dominant weight loss at $\sim 97^{\circ} \mathrm{C}$, associated with gypsum.

\section{Analyses of degraded samples - ATR-FTIR measurements} The results of the ATR-FTIR measurements were in agreement with the XRD data. They confirmed three major chemical compounds in TSRC-1 - carbonates at $1482 \mathrm{~cm}^{-1}$ and $868 \mathrm{~cm}^{-1}$, silica gel at $1228 \mathrm{~cm}^{-1}$ and C,N-A-S hydrates at $986 \mathrm{~cm}^{-1}$ and $903 \mathrm{~cm}^{-1}$. The spectrum of the core sample was similar to the control, while the chemical composition of the surface layer mostly consisted of some major sulfate phase, and minor C,N-A-S hydrates along with silica gel. An increased fly ash content (TSRC-3) (Figure 6) engendered a shift of the most prominent $\mathrm{M}-\mathrm{O}$ (M: silicon or aluminium) asymmetric ( $\left.V_{\text {as M-O }}\right)$ band of C,N-A-S hydrates to a higher frequency, from $903 \mathrm{~cm}^{-1}$ for TSRC-1 to $989 \mathrm{~cm}^{-1}$ for TSRC-3, and the absence of a silica-gel-related band around $1220 \mathrm{~cm}^{-1}$. The major bands of the TSRC-3 components were at $1034 \mathrm{~cm}^{-1}$ from $V_{\text {as M-O }}$ stretching in $\mathrm{Al}_{2} \mathrm{O}_{3}-\mathrm{SiO}_{2}$ in FAF and at $770 \mathrm{~cm}^{-1}$ for aluminiumoxygen in the 'condensed' aluminate $\left(\mathrm{AlO}_{4}\right)$ tetrahedra network 

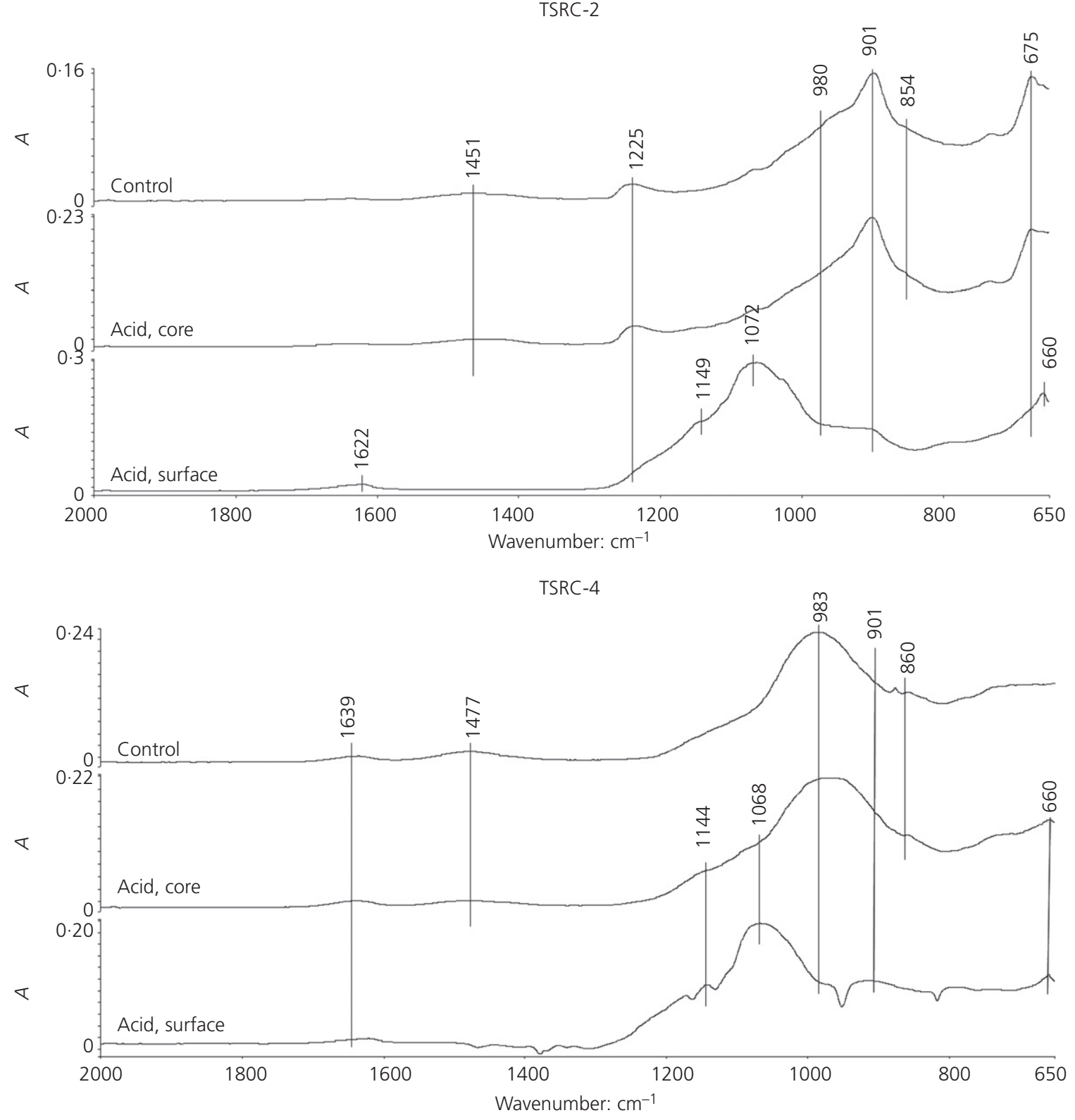

Figure 7. FTIR spectra of TSRC-2 and TSRC-4 blends before and after sulfuric acid exposure)

of the monocalcium aluminate phase of CAC (not shown) (Husung and Doremus, 1990; Roy, 1987). Thus, the shift of the TSRC-3 band to higher frequency points to a larger proportion of FAF reaction products with lower calcium content. FTIR analyses of the acid-exposed TSRC-3 confirmed the presence of sulfate in the core, with shoulder and weak bands at 1159, 1074 and $660 \mathrm{~cm}^{-1}$. As expected, the surface layer of this cement was dominated by sulfates, while the presence of C,N-A-S hydrates was very low, if any.

The spectra of blends TSRC-2 and TSRC-4 (Figure 7) were very similar to those of TSRC-1 and 3, respectively.
The spectrum of the control CPC (Figure 8) included bands of groups containing phosphorous, alumosilicates and silica gel. They were $\mathrm{P}-\mathrm{O}$ asymmetric $\left(V_{\text {as }} \mathrm{P}-\mathrm{O}\right)$ stretching vibration bands at 1066 and $1031 \mathrm{~cm}^{-1}$ and $\mathrm{P}-\mathrm{O}$ symmetric $\left(V_{\mathrm{s}-\mathrm{P}-\mathrm{O}}\right)$ stretching at $905 \mathrm{~cm}^{-1}$ of phosphates $\left(\mathrm{PO}_{4}{ }^{3-}\right)$ in calcium phosphates (Kolmas et al., 2015; Low et al., 2011; Nasiri-Tabrizi and Fahami, 2013; Taddei et al., 2014). The band at $905 \mathrm{~cm}^{-1}$ was also attributable to the asymmetric ( $\left.V_{\text {as M-O }}\right)$ stretching mode (M: silicon or aluminium) in the $\mathrm{Si}-\mathrm{O}-\mathrm{Si}$ and $\mathrm{Si}-\mathrm{O}-\mathrm{Al}$ in $\mathrm{C}$, $\mathrm{N}-\mathrm{A}-\mathrm{S}$ reaction products and that at $676 \mathrm{~cm}^{-1}$ was interpreted as the $\mathrm{M}-\mathrm{O}$ symmetric $\left(V_{\mathrm{s} \text { M-O }}\right)$ stretching mode (Clayden et al., 1999; Criado et al., 2007; Husung and Doremus, 1990; 

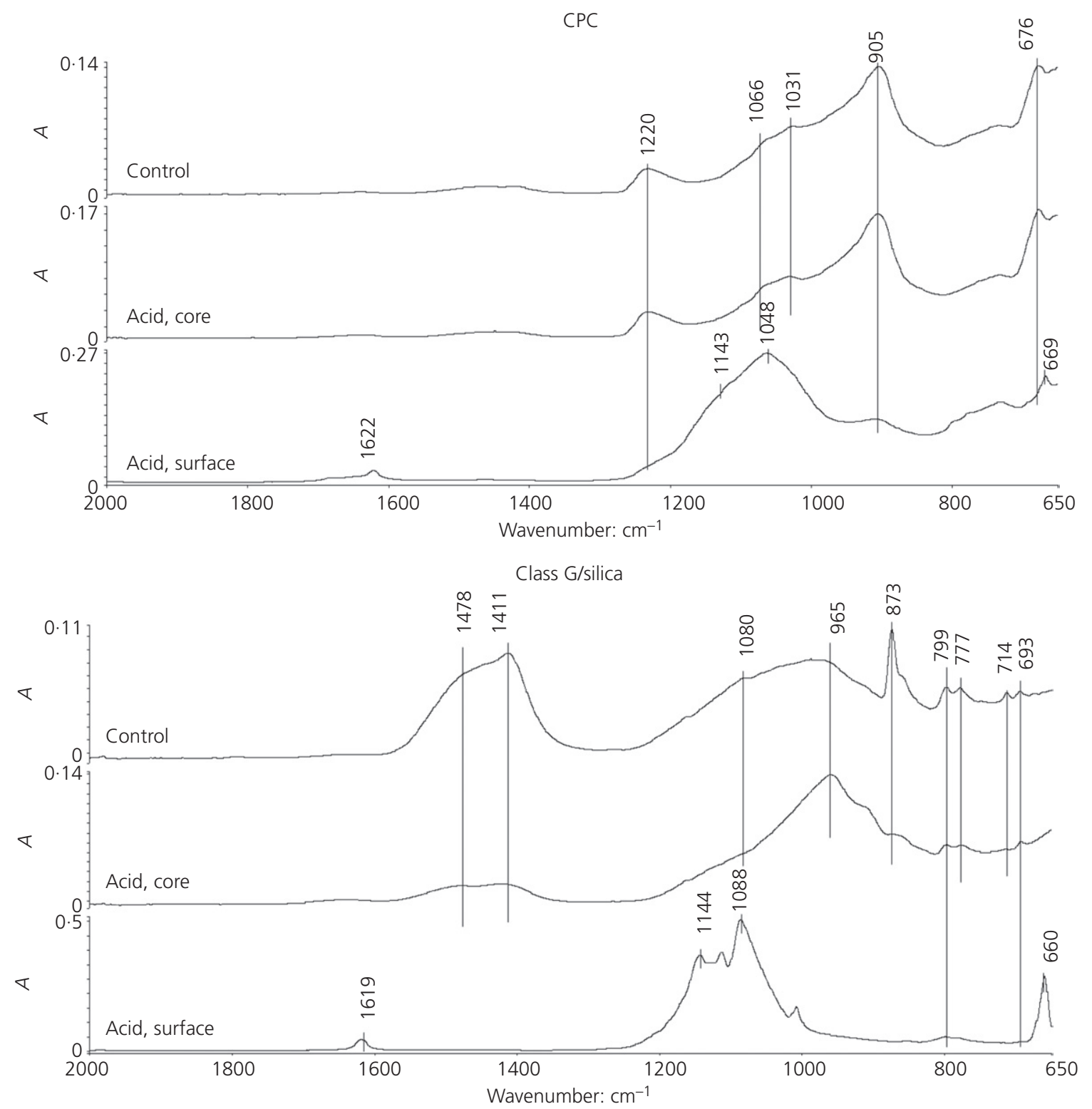

Figure 8. FTIR spectra of CPC and class G/silica blends before and after sulfuric acid exposure

Roy, 1987; Sugama et al., 2000) and a band of silica gel at $1220 \mathrm{~cm}^{-1}$ (Almeida and Pantano, 1990; Donatello et al., 2013; Fidalogo and Ilharco, 2001; Innocenzi, 2003; Tanner et al., 2000). The spectrum of the sample core after acid exposure resembled the unexposed sample. The surface of the CPC was primarily composed of sulfates; the presence of weak bands at $1220 \mathrm{~cm}^{-1}$ and $905 \mathrm{~cm}^{-1}$ suggested some silica gel, C,N-A-S and possibly some calcium phosphate coexisting with sulfates.

The spectrum of the control class G/silica blend (Figure 8) included two bands at $1478 \mathrm{~cm}^{-1}$ and $1411 \mathrm{~cm}^{-1}$ from the $\mathrm{C}-\mathrm{O}$ asymmetric ( $\left.V_{\text {as C-O }}\right)$ stretching vibration in carbonate
$\left(\mathrm{CO}_{3}{ }^{2-}\right)$ and the band at $873 \mathrm{~cm}^{-1}$ from $\mathrm{O}-\mathrm{C}-\mathrm{O}$ out-of-plane bending $\left(\delta_{\mathrm{O}-\mathrm{C}-\mathrm{O}}\right)$ in carbonate (Gunasekaran et al., 2006; Xyla and Koutsoukos, 1989; Ylmen and Jaglid, 2013), the calciumsilicate-hydrate-related $\mathrm{Si}-\mathrm{O}$ asymmetric stretching $\left(V_{\text {as }} \mathrm{Si-O}\right)$ in the silicate $\left(\mathrm{SiO}_{4}{ }^{-4}\right)$ wide band at $965 \mathrm{~cm}^{-1}$ (Mollah et al., 2000; Taddei et al., 2014) and the three quartz-related bands at 1080, 799 and $693 \mathrm{~cm}^{-1}$ (Guan et al., 2014; Ramasamy and Suresh, 2009; Saikia et al., 2008). The spectrum from the core of the acid-exposed sample was similar to that of the control. In agreement with XRD and TGA analyses, the surface sample revealed replacement of the most of the carbonate- and silicate-associated bands by the three sulfate ones $\left(\mathrm{SO}_{4}{ }^{2-}\right)$, at 1144, 1088 and $660 \mathrm{~cm}^{-1}$, in gypsum (Al-Hosney and 
Advances in Cement Research

Volume 28 Issue 7
Acid resistance of calcium aluminate

cement-fly ash $\mathrm{F}$ blends

Pyatina and Sugama

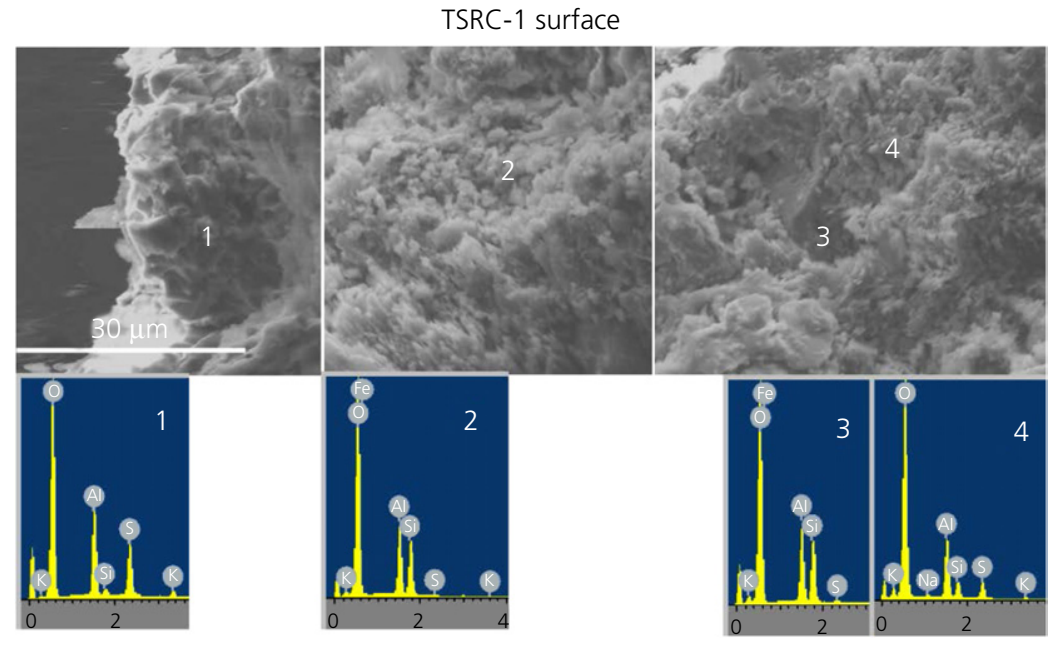

TSRC-1 core

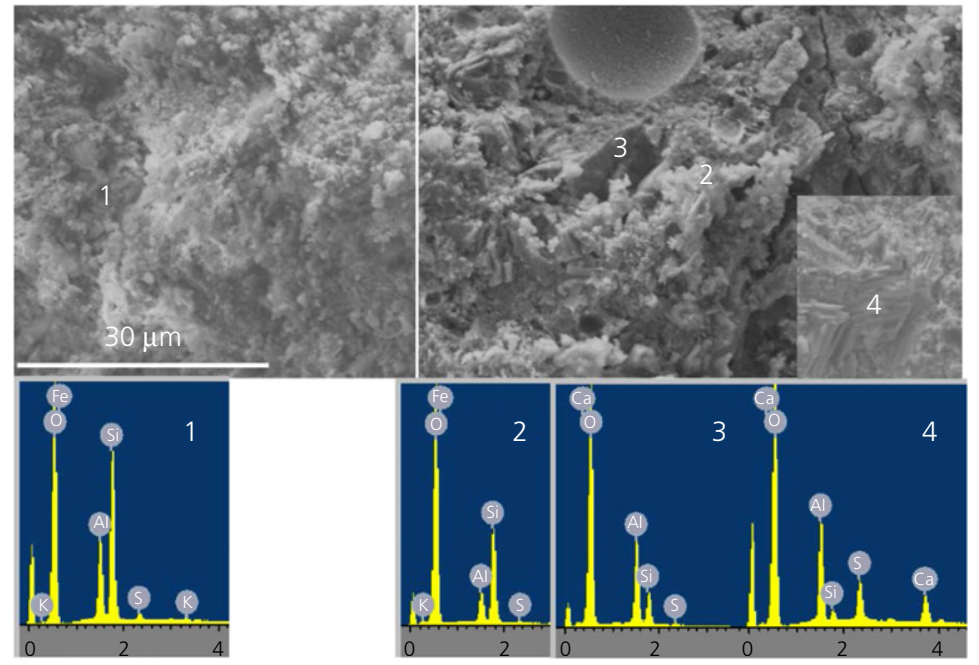

TSRC-2 surface

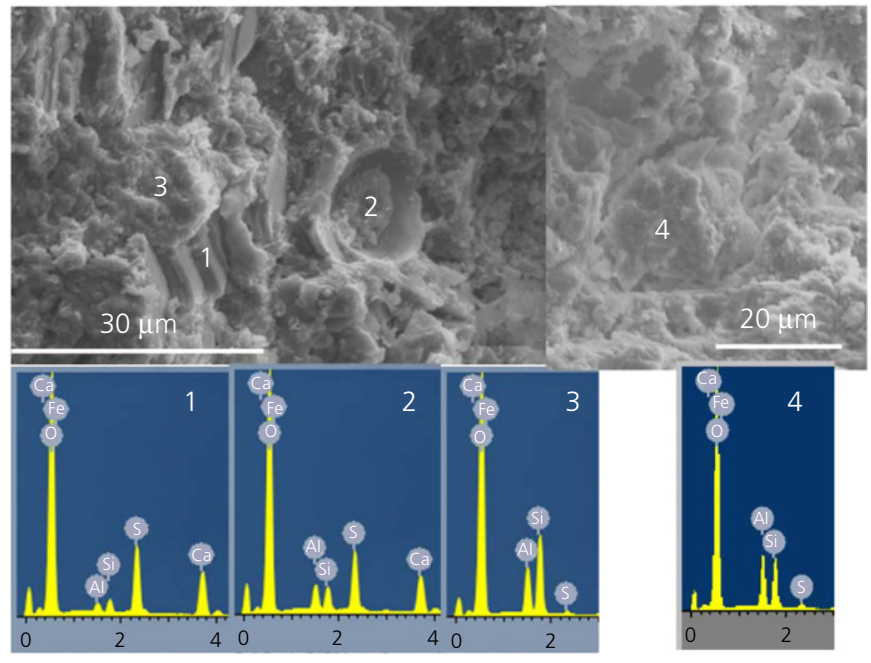

Figure 9. SEM images of typical cement microstructures and their elemental compositions after sulfuric acid exposure 
Grassian, 2005; García-Lodeiro et al., 2011; Ylmen et al., 2009).

\section{Analyses of degraded samples - morphologies of TSRC-1} and TSRC-2

The microphotograph of the acid-treated TSRC-1 sample shows a rough, partially eroded surface (Figure 9(a)) rich in aluminium and sulfur with small inclusions of alunite (site 1) (Stoffregen et al., 1994) and sites with the elemental composition of mullite (site 2). A typical atomic composition of the mostly amorphous surface is similar to the alunite elemental composition (site 3 ), in agreement with other data. The core of the sample is dense (Figure 9(b), site 1), amorphous for the most part, with some aluminium-silicate crystals (sites 2 and 3) and prints of displaced FAF particles. It becomes more porous and less compact closer to the surface. The insert on the microphotograph shows some sulfur-containing crystals with a strong aluminium signal (site 4). This could be an intermediate product of cement-sulfuric acid interactions that later decomposes with the loss of calcium to sulfate. Similarly, the microphotograph of sample TSRC-2 shows an eroded rough surface (Figure 9(c)). The bassanite crystals are either embedded into the sample's matrix (site 1) or grow in the open shells of the partially reacted FAF particles (site 2). For the most part, the surface is composed of silica-aluminate gel (site 3) with some sites that have a typical composition of dmisteinbergite crystals (site 4). The core structure of the sample is porous, mostly amorphous with inclusions of some katoite crystals (site 1) and partially reacted FAF particles (site 3). The SEM data confirmed that the surfaces of samples TSRC-1 and TSRC-2 were attacked by the sulfuric acid, resulting in the formation of amorphous and crystalline sulfate-containing products, some surface erosion and increased porosity.

\section{Conclusions}

Blends of thermal shock resistant cement (TSRC) composed of calcium aluminate cement (CAC) and class F fly ash (FAF) activated with sodium metasilicate were hydrated for $24 \mathrm{~h}$ at $300^{\circ} \mathrm{C}$ and tested for their resistance to sulfuric acid solutions at $90^{\circ} \mathrm{C}$ for $18 \mathrm{~d}$. Four blends were prepared with CAC of varied calcium and aluminium contents and different $\mathrm{CAC}$ to fly ash ratios. Their short-term resistance to acid attack was compared to that of a Portland cement class G/silica blend and to a calcium phosphate cement (CPC) made of CAC, FAF and sodium hexametaphosphate.

The following general conclusions can be drawn from the study.

(a) After $18 \mathrm{~d}$ of sulfuric acid exposure at $90^{\circ} \mathrm{C}$, all the TSRC formulations lost weight and decreased in diameter while keeping or increasing their compressive strength and forming only a thin rim layer. The changes were the smallest for the aluminium-rich blend (TSRC-1) and the most significant for the silicon- and calcium-rich blend
(TSRC-4). Of all the tested formulations, the CPC lost most weight, diameter and some compressive strength. In contrast, the class $\mathrm{G} / \mathrm{silica}$ blend increased both in mass and diameter due to the formation of a thick gypsum layer partially eroded from the core of the sample.

(b) The attack of sulfuric acid on TSRC samples starts with calcium-sulfate reactions and calcium removal from the samples' surfaces. All calcium-containing hydrates were susceptible to the sulfuric acid attack. These hydrates included feldspar family minerals such as anorthite and dmisteinbergite and various zeolites formed in pozzolanic reactions of the fly ash. Aluminium formed potassiumaluminium hydrate, alunite, which persisted under the experimental conditions. The aluminium-containing CAC hydration product, boehmite, better resisted acid attack than did aluminium-containing phases formed from FAF (zeolites). XRD and $\mu$ EDX data confirmed boehmite's survival in the surface layers of aluminium-rich blends and the lesser surface loss of aluminium compared with the blends rich in fly ash.

(c) In the CPC, the hydration products containing calcium phosphate were susceptible to attack by the sulfuric acid. The phases containing phosphates, such as apatites and calcium phosphate, reacted with the sulfuric acid, resulting in the formation of gypsum that helped to prevent sulfur penetrating into the core of the samples. However, it could not stop mass, diameter and compressive strength losses. In agreement with numerous previous studies, the class $\mathrm{G} /$ silica blend hydrates reacted with the sulfate ions, with the formation of a thick gypsum layer. Concentrations of both calcium and silicon strongly decreased in the surface layer of this sample and its integrity was compromised due to the partial erosion of calcium sulfate and low cohesion of the remaining silica gel.

(d) Based on sulfur penetration into the core of the samples, changes in sample appearance, size and compressive strength, the resistance of the tested blends to short-term exposure to $\mathrm{pH} 0.5$ sulfuric acid at $90^{\circ} \mathrm{C}$ could be ranked as follows: TSRCs rich in aluminium and CAC (TSRC-1 and TSRC-2) $>$ TSRCs rich in fly ash (TSRC-3 and TSRC-4) > CPC > class G/silica blend. Long-term exposure tests would be necessary to confirm the long-term stability of the alunite phase formed in aluminium-rich formulations as a result of sulfuric acid attack. The CAC-FAF blends with high cement content ( $60 \%$ by weight) were the most resistant under the experimental conditions and would thus be the preferred choice for the acidic environments of geothermal wells.

\section{Acknowledgements}

This publication was based on work supported by the Geothermal Technologies Office in the US Department of Energy (DOE) Office of Energy Efficiency and Renewable Energy (EERE), under the auspices of the US DOE, 
Washington, DC, under contract DE-AC02-98CH 10886. The research was carried out in part at the Center for Functional Nanomaterials, Brookhaven National Laboratory, which is supported by the US Department of Energy, Office of Basic Energy Sciences, under contract DE-SC0012704.

\section{REFERENCES}

Al-Akhras NM (2006) Durability of metakaolin concrete to sulfate attack. Cement and Concrete Research 36(9): 1727-1734.

Alexander MG, Bertron A and De Belie N (2013) Performance of Cement-Based Materials in Aggressive Aqueous Environments. Springer, Dordrecht, the Netherlands, State-of-the-Art Report, RILEM TC 211-PAE.

Al-Hosney HA and Grassian VH (2005) Water, sulfate dioxide and nitric acid adsorption on calcium carbonate: a transmission and ATR-FTIR study. Physical Chemistry Chemical Physics 7(6): 1266-1276.

Allahverdi A and Skvara F (2001) Nitric acid attack on hardened paste of geopolymeric cements - Part 2. Ceramics-Silikaty 45(4): 143-149.

Allahverdi A and Skvara F (2005) Sulfuric acid attack on hardened paste of geopolymer cements Part 1. Mechanism of corrosion at relatively high concentrations. CeramicsSilikaty 49(4): 225-229.

Almeida RM and Pantano CG (1990) Structure investigation of silica gel films by infrared spectroscopy. Journal of Applied Physics 68(8): 4225-4232.

Bakharev T (2005) Resistance of geopolymer materials to acid attack. Cement and Concrete Research 36(4): 658-670.

Bakharev T, Sanjayan JG and Cheng YB (2002) Sulfate attack on alkali-activated slag concrete. Cement and Concrete Research 32(2): 211-216.

Beddoe R and Dorner HW (2005) Modelling acid attack on concrete: part I. The essential mechanisms. Cement and Concrete Research 35(12): 2333-2339.

Bentz DP and Garboczi EJ (1992) Modeling the leaching of calcium hydroxide from cement paste: effects on pore space percolation and diffusivity. Materials and Structures 25(9): 523-533.

Chen JJ, Thomas JJ, Taylor HFW and Jennings HM (2004) Solubility and structure of calcium silicate hydrate. Cement and Concrete Research 34(9): 1499-1519.

Chindaprasirt P, Paisitsrisawat P and Rattanasak U (2014) Strength and resistance to sulfate and sulfuric acid of ground fluidized bed combustion fly ash-silica fume alkali-activated composite. Advanced Powder Technology 25(3): 1087-1093.

Clayden NJ, Esposito S, Aronne A and Pernice P (1999) Solid state 27Al NMR and FTIR study of lanthanum aluminosilicate glasses. Journal of Non-Crystalline Solids 258(1-3): 11-19.

Criado M, Fernandez-Jimenez A and Palomo A (2007) Alkali activation of fly ash: effect of the $\mathrm{SiO}_{2} / \mathrm{Na}_{2} \mathrm{O}$ ratio part I:
FTIR study. Microporous and Mesoporous Materials 106(1): 180-191.

Donatello S, Palomo A and Fernandez-Jimenez A (2013) Durability of very high volume fly ash cement pastes and mortars in aggressive solutions. Cement \& Concrete Composites 38(1): 12-20.

Fidalogo A and Ilharco LM (2001) The defect structure of sol-gel-derived silica/polytetrahydrofuran hybrid films by FTIR. Journal of Non-Crystalline Solids 283(1): 144-154.

Foldvari M (2011) Handbook of Thermogravimetric System of Minerals and its Use in Geological Practice. Geological Institute of Hungary, Budapest, Hungary, Occasional Papers, vol. 213.

García-Lodeiro I, Palomo A, Fernández-Jimenez A and Macphee DE (2011) Compatibility studies between $\mathrm{N}-\mathrm{A}-\mathrm{S}-\mathrm{H}$ and C-A-S-H gels. Study in the ternary diagram $\mathrm{Na}_{2} \mathrm{O}-\mathrm{CaO}-\mathrm{Al}_{2} \mathrm{O}_{3}-\mathrm{SiO}_{2}-\mathrm{H}_{2} \mathrm{O}$. Cement and Concrete Research 41(9): 923-931.

Glasser FP, Marchand J and Samson E (2008) Durability of concrete - degradation phenomena involving detrimental chemical reactions. Cement and Concrete Research 38(2): 226-246.

Guan W, Ji F, Fang Z et al. (2014) Low hydrothermal temperature synthesis of porous calcium silicate hydrate with enhanced reactivity $\mathrm{SiO}_{2}$. Ceramics International 40(3): 4415-4420.

Gunasekaran S, Anbalagan G and Pandi S (2006) Raman and infrared spectra of carbonate of calcite structure. Journal of Raman Spectroscopy 37(9): 892-899.

Gutberlet T, Hilbig $\mathrm{H}$ and Beddoe RE (2015) Acid attack on hydrated cement - effect of mineral acids on the degradation process. Cement and Concrete Research 74: 35-43.

Haga K, Shibata M, Hironaga M, Tanaka S and Nagasaki S (2002) Silicate anion structural change in calcium silicate hydrate gel on dissolution of hydrated cement. Journal of Nuclear Science Technology 39(5): 540-547.

Harada K, Tanaka K and Nagashima K (1972) New data on the analcime wairakite series. American Mineralogist 57(5-6): 924-931.

Husung RD and Doremus RH (1990) The infrared transmission spectra of four silicate glasses before and after exposure to water. Journal of Materials Research 5(10): 2209-2216.

Innocenzi P (2003) Infrared spectroscopy of sol-gel derived silica-based films: a spectra-microstructure overview. Journal of Non-Crystalline Solids 316(2-3): 309-319.

Kandasamy S and Shehata MH (2014) Durability of ternary blends containing high calcium fly ash and slag against sodium sulphate attack. Construction and Building Materials 53: 267-272.

Kiyosaki J, Tanaka K, Taguchi S et al. (2003) Subsurface high temperature hypogene acid alteration at the Hatchobaru geothermal field, Kyushu, Japan. Proceedings of the 25th New Zealand Geothermal Workshop, Auckland, 
New Zealand. NZGA, Auckland, New Zealand, pp. 161-165.

Kolmas J, Kaflak A, Zima A and Slosarczyk A (2015)

Alpha-tricalcium phosphate synthesized by two different routes: structural and spectroscopic characterization. Ceramics International 41(4): 5727-5733.

Kudowski W (2004) The protective layer and decalcification of $\mathrm{C}-\mathrm{S}-\mathrm{H}$ in the mechanism of chloride corrosion of cement paste. Cement and Concrete Research 34(9): 1555-1559.

Lamberet S, Guinot D, Lempereur E, Talley J and Alt C (2008)

Field investigations of high performance calcium aluminate mortar for wastewater applications. In Calcium Aluminate Cements: Proceedings of the 2008 Centenary Conference (Fentiman C, Mangabhai R and Scrivener K (eds)). IHS BRE Press, Bracknell, UK, pp. 269-277.

Larreur-Cayol S, Bertron A and Escadeillas G (2011) Degradation of cement-based materials by various organic acids in agro-industrial waste-waters. Cement and Concrete Research 41(8): 882-892.

Lee ST, Moon HY and Swamy RN (2005) Sulfate attack and role of silica fume in resisting strength loss. Cement \& Concrete Composites 27(1): 65-76.

Lichti KA and Yanagisawa N (2015) Geothermal energy materials and process issues. Proceedings of the World Geothermal Congress, Melbourne, Australia. WGC, Melbourne, Australia, pp. 1-8.

Low KL, Zein SHS, Tan SH, McPhail DS and Boccaccini AR (2011) The effect of interfacial bonding of calcium phosphate cements containing bio-mineralized multi-walled carbon nanotube and bovine serum albumin on the mechanical properties of calcium phosphate cements. Ceramics International 37(7): 2429-2435.

Miller JL, Elwood Madden AS, Phillips-Lander CM, Pritchett BN and Elwood Madden ME (2016) Alunite dissolution rates: dissolution mechanisms and implications for Mars. Geochimica et Cosmochimica Acta 172: 93-106.

Mollah MYA, Yu W, Schennach R and Cocke DL (2000) A Fourier transform infrared spectroscopic investigation of the early hydration of Portland cement and the influence of sodium lignosulfonate. Cement and Concrete Research 30(2): 267-273.

Nasiri-Tabrizi B and Fahami A (2013) Mechanochemical synthesis and structural characterization of nano-sized amorphous tricalcium phosphate. Ceramics International 39(8): 8657-8666.

Nuruddeen MM (2014) Thermal analysis of cement paste partially replaced with Neem Seed Husk Ash. International Journal of Scientific and Engineering Research 5(1): $1101-1105$

Ouyang C, Nanni A and Chang WF (1988) Internal and external sources of sulfate ions in Portland cement mortar: two types of chemical attack. Cement and Concrete Research 18(5): 699-709.
Puertas F, Goni S, Hernandez MS, Varga C and Guerrero A (2012) Comparative study of accelerated decalcification process among $\mathrm{C}_{3} \mathrm{~S}$, grey and white cement pastes. Cement and Concrete Composites 34(3): 384-391.

Pyatina T and Sugama T (2016) Resistance of fly ash-Portland cement blends to thermal shock. Advances in Cement Research 28(2): 121-131, http://dx.doi.org/10.1680/adcr. 15.00030.

Ramasamy V and Suresh G (2009) Mineral characterization and crystalline nature of quartz in Ponnaiyar river sediments, Tamilnadu, India. American-Eurasian Journal of Science Research 4(2): 103-107.

Roy BN (1987) Spectroscopic analysis of the structure of silicate glasses along the joint of $\mathrm{xMAlO}_{2}-(1-\mathrm{x}) \mathrm{SiO}_{2}(\mathrm{M}=\mathrm{Li}, \mathrm{Na}$, $\mathrm{K}, \mathrm{Rb}, \mathrm{Cs})$. Journal of the American Ceramic Society 70(3): 183-192.

Saikia BJ, Parthasarathy G and Sarmah NC (2008) Fourier transform infrared spectroscopic estimation of crystallinity in $\mathrm{SiO}_{2}$ based rocks. Bulletin of Materials Science 31(5): $775-779$.

Steiner A (1968) Clay minerals in hydrothermally altered rocks at Wairakei, New Zealand. Clays and Clay Minerals 16(3): 193-213.

Stoffregen R, Rye RO and Wasserman MD (1994) Experimental studies of alunite: II. Rates of alunite-water alkali and isotope exchange. Geochimica et Cosmochimica Acta 58(2): 917-929.

Sugama T (1997) Hydrothermal treatment of calcium aluminate fly ash sodium metaphosphate cements. Advances in Cement Research 9(34): 65-73, http://dx.doi. org/10.1680/adcr.1997.9.34.65.

Sugama T, Weber L and Brothers LE (1999) Resistance of sodium polyphosphate-modified fly ash/calcium aluminate blend cements to hot $\mathrm{H}_{2} \mathrm{SO}_{4}$ solution. Cement and Concrete Research 29(12): 1969-1976.

Sugama T, Weber L and Brothers LE (2000) Sodiumpolyphosphate-modified fly ash/calcium aluminate blend cement: durability in wet, harsh geothermal environments. Materials Letters 44(1): 45-53.

Sugama T, Pyatina T and Gill S (2012) Thermal Shock Resistant Cement. Brookhaven National Laboratory, Upton, NY, USA, Report BNL-101087-2012-IR.

Taddei P, Modena E, Tinti A et al. (2014) Effect of the fluoride content on the bioactivity of calcium silicate-based endodontic cements. Ceramics International 40(3): 4095-4107.

Tanner PA, Yan B and Zhang H (2000) Preparation and luminescence properties of sol-gel hybrid materials incorporated with europium complexes. Journal of Materials Science 35(17): 4325-4328.

Villasenor LB and Vicedo RO (2010) Exclusion of acid sulfate fluid in wells at Tiwi Geothermal field, Albay Province, Philippines. Proceedings of the World Geothermal Congress, Bali, Indonesia. 
Winnefeld F, Leemann A, Lucuk M, Svoboda P and Neuroth M (2010) Assessment of phase formation in alkali activated low and high calcium fly ashes in building materials. Construction and Building Materials 24(6): 1086-1093.

Wohletz K and Grant H (1992) Volcanology and Geothermal Energy. University of California Press, Berkeley, CA, USA. Xyla AG and Koutsoukos PG (1989) Quantitative analysis of calcium carbonate polymorphs by infrared spectroscopy. Journal of the Chemical Society, Faraday Transactions 1(85): 3165-3172.

Ylmen R and Jaglid U (2013) Carbonation of Portland cement studied by diffuse reflection Fourier transform infrared spectroscopy. International Journal of Concrete Structures and Materials 7(1): 119-125.

YImen R, Jaglid U, Steenari BM and Panas IN (2009) Early hydration and setting of Portland cement monitored by IR, SEM and Vicas techniques. Cement and Concrete Research 39(5): 433-439.

Zivica V (2004) Acidic attack of cement based materials - a review part 3: research and test methods. Construction and Building Materials 18(9): 683-688.

Zivica V and Bajza A (2002) Acidic attack of cement-based materials - a review part 2. Factors of rate of acidic attack and protective measures. Construction and Building Materials 16(4): 215-222.

\section{WHAT DO YOU THINK?}

To discuss this paper, please submit up to 500 words to the editor at journals@ice.org.uk. Your contribution will be forwarded to the author(s) for a reply and, if considered appropriate by the editorial panel, will be published as a discussion in a future issue of the journal. 